\title{
Flow control over an airfoil using virtual Gurney flaps
}

\author{
Li-Hao Feng ${ }^{1}$, Kwing-So Choi ${ }^{2} \uparrow$ and Jin-Jun Wang ${ }^{1}$ \\ ${ }^{1}$ Fluid Mechanics Key Laboratory of Education Ministry, Beijing University of Aeronautics and \\ Astronautics, Beijing 100191, China \\ ${ }^{2}$ Faculty of Engineering, University of Nottingham, Nottingham NG7 2RD, UK
}

(Received 23 May 2014; revised 11 November 2014; accepted 8 January 2015; first published online 20 February 2015)

Flow control over a NACA 0012 airfoil is carried out using a dielectric barrier discharge (DBD) plasma actuator at the Reynolds number of 20000 . Here, the plasma actuator is placed over the pressure (lower) side of the airfoil near the trailing edge, which produces a wall jet against the free stream. This reverse flow creates a quasi-steady recirculation region, reducing the velocity over the pressure side of the airfoil. On the other hand, the air over the suction (upper) side of the airfoil is drawn by the recirculation, increasing its velocity. Measured phase-averaged vorticity and velocity fields also indicate that the recirculation region created by the plasma actuator over the pressure surface modifies the near-wake dynamics. These flow modifications around the airfoil lead to an increase in the lift coefficient, which is similar to the effect of a mechanical Gurney flap. This configuration of DBD plasma actuators, which is investigated for the first time in this study, is therefore called a virtual Gurney flap. The purpose of this investigation is to understand the mechanism of lift enhancement by virtual Gurney flaps by carefully studying the global flow behaviour over the airfoil. First, the recirculation region draws the air from the suction surface around the trailing edge. The upper shear layer then interacts with the opposite-signed shear layer from the pressure surface, creating a stronger vortex shedding from the airfoil. Secondly, the recirculation region created by a DBD plasma actuator over the pressure surface displaces the positive shear layer away from the airfoil, thereby shifting the near-wake region downwards. The virtual Gurney flap also changes the dynamics of laminar separation bubbles and associated vortical structures by accelerating laminar-to-turbulent transition through the Kelvin-Helmholtz instability mechanism. In particular, the separation point and the start of transition are advanced. The reattachment point also moves upstream with plasma control, although it is slightly delayed at a large angle of attack.

Key words: boundary layer control, boundary layer separation, boundary layer stability

$†$ Email address for correspondence: kwing-so.choi@nottingham.ac.uk 


\section{Introduction}

Low-Reynolds-number aerodynamics has attracted much interest recently with the deployment of micro air vehicles (MAVs) and small unmanned aerial vehicles (UAVs) in a variety of commercial and military missions (Mueller \& Delaurier 2003). However, the airfoil aerodynamics at low Reynolds numbers is still not well understood, mainly due to the laminar separation bubbles during flow separation, whose behaviour is strongly dependent on the Reynolds number (O'Meara \& Mueller 1987). Airfoils operated at low Reynolds numbers incur an early flow separation with the formation of separation bubbles (Ellsworth \& Mueller 1991). Laminar separation bubbles can burst with only a small increase in the angle of attack, leading to a sudden drop in the lift (Zhou et al. 2011). It has also been shown that the lift coefficient does not increase linearly even with small angle of attack (Laitone 1997; Kim, Chang \& Chung 2011).

\subsection{Laminar separation bubbles}

A pioneering study of separation bubbles over a flat plate under an adverse pressure gradient was conducted by Gaster (1967), who indicated that the structure of the bubble depended on the Reynolds number of the separating boundary layer and a parameter based on the pressure rise over the region occupied by the bubble. This work was followed by Horton (1969), who provided a simple pressure gradient criterion for the laminar separation bubble problem to determine a condition for the reattachment of a turbulent shear flow. Pauley, Moin \& Reynolds (1990) obtained time-accurate solutions of the Navier-Stokes equations, where they found that a strong adverse pressure gradient created periodic vortex shedding from the separation region. Watmuff (1999) showed that the dispersion and rapid growth associated with the Kelvin-Helmholtz $(\mathrm{K}-\mathrm{H})$ wavepacket led to the formation of rolled-up structures, which ultimately evolved into large-scale vortex loops in the vicinity of the reattachment. Alam \& Sandham (2000) conducted a direct numerical simulation (DNS) of short laminar separation bubbles with turbulent reattachment, where they found that the separated shear layer underwent transition via oblique modes and $\Lambda$-vortex-induced breakdown. Spalart \& Strelets (2000) suggested that the transition mechanism involved the wavering of the shear layer and the $\mathrm{K}-\mathrm{H}$ vortices. Subsequently, Diwan \& Ramesh (2009) observed an exponential growth of the disturbance in the region upstream of the mean maximum height of the bubble.

Batill \& Muller (1981) studied the free shear layer associated with the laminar separation bubble over a NACA 663-018 airfoil at the Reynolds numbers of 50000-120000, showing that the transition process in the free shear layer was indeed three-dimensional. Lian \& Shyy (2007) used a Reynolds-averaged transition model to study the laminar boundary layer transition and separation over an SD7003 airfoil at Reynolds numbers between 40000 and 200000, where they found that the Tollmien-Schlichting (T-S) wave was amplified under a strong pressure gradient to expedite the boundary transition. Yang \& $\mathrm{Hu}$ (2008) studied the behaviour of flow over a NASA LS(1)-0417 airfoil at the Reynolds number of 70000 , where the separated laminar boundary layer reattached as a turbulent flow when the angle of attack exceeded $8^{\circ}$. The laminar separation bubbles moved upstream with an increase in the angle of attack, but their length remained nearly constant. Jones, Sandberg \& Sandham (2010) carried out a DNS study of the flow over a NACA 0012 airfoil at the Reynolds number of 50000 to show that the separation bubble exhibited no absolute instability. Based on a large eddy simulation (LES) study over a NACA 
0012 airfoil at the Reynolds number of 50000, Almutairi, Jones \& Sandham (2010) found an intermittent bursting of laminar separation bubbles near stall, to form either a long bubble or to lead to a fully separated flow. Using a novel combination of flow visualization, hot-wire measurements and surface pressure measurements, Boutilier \& Yarusevych (2012) showed that the disturbance convection speeds in the separation bubble were between $30 \%$ and $50 \%$ of the boundary layer edge velocity.

These investigations seem to suggest that the formation and development of separation bubbles over airfoils are similar to those over flat plates under pressure gradients. However, only limited results are currently available on the relationship between the development of separation bubbles and the shedding of vortices from airfoils. Yarusevych, Sullivan \& Kawall (2009) showed that the length scale of the wake vortices decreased significantly and the vortex pattern became less organized when separation bubbles were formed over the airfoil. Huang \& Lin (1995), Yarusevych, Sullivan \& Kawall (2006) and Yarusevych \& Boutilier (2011) investigated the turbulent wake development of NACA four-digit series airfoils at low Reynolds numbers, and demonstrated that the dimensionless vortex shedding frequency increased linearly with the Reynolds number. Hain, Kähler \& Radespiel (2009) showed that the $\mathrm{K}-\mathrm{H}$ instability led to a spanwise vortex formation in the shear layer above the separation bubble over an SD7003 aerofoil at the Reynolds number of $R e=66000$. Burgmann, Brücker \& Schröder (2006) investigated a similar flow by using scanning particle image velocimetry (PIV), where they observed the quasi-periodic development of large vortex rolls at the downstream end of the separation bubble. Using time-resolved PIV and volumetric PIV measurements, Burgmann, Dannemann \& Schröder (2008) further discussed the effects of the K-H instability on a transitional separation bubble and the temporal and spatial evolution of vortical structures. In a similar experiment, Burgmann \& Schröder (2008) investigated the influence of the angle of attack, Reynolds number and free-stream turbulence level on the size, structure and characteristics of laminar separation bubbles.

\subsection{Flow separation control}

Flow separation control is an effective way to improve the aerodynamic performance of airfoils. For example, the lift can be increased and the drag can be reduced by delaying or eliminating flow separation. Greenblatt \& Wygnanski (2000) presented a comprehensive review of flow separation control by periodic excitation. In particular, they suggested that the reduced frequency of the effective excitation should be in the range of $0.3-4$ with the momentum coefficient of between $0.01 \%$ and $3 \%$. Wu et al. (1998) carried out a Reynolds-averaged two-dimensional simulation to show that a massively separated flow over a NACA 0012 airfoil could be effectively controlled by a periodic blowing and suction near the leading edge. It was demonstrated that the unsteady forcing modulated the evolution of the separated shear layer by promoting the formation of vortices, which favourably interacted with trailing-edge vortices. Atik et al. (2005) used suction near the leading edge as a means of controlling flow separation to inhibit dynamic stall. Substantial delay in separation was achieved even when the suction strength was weak, provided that it was initiated at an early stage. Duvigneau, Hay \& Visonneau (2007) used a synthetic jet to control post-stall flow over a NACA 0012 airfoil, and demonstrated that a lift increment up to $57 \%$ could be obtained. Genç, Kaynak \& Yapici (2011) carried out flow control of a NACA 2415 airfoil at the Reynolds number of 200000 , where the separation bubble was eliminated by simultaneous blowing and suction, resulting in a marginal lift increase and drag 
reduction. Wahidi \& Bridges (2012) used distributed suction over an LA2573a airfoil at Reynolds numbers of 150000 and 250000 , shifting the separation and transition points further downstream.

Nickerson (1986) used vane-type vortex generators to control flow over a NACA 0024 airfoil at Reynolds numbers of 100000-200000. Wind tunnel tests indicated that the stall angle of the airfoil was increased by the passive device, although the maximum lift was slightly reduced. Seshagiri, Cooper \& Traub (2009) conducted a similar test using vortex generators at the Reynolds numbers of 80000-160000. The force measurement indicated that the vortex generators increased the maximum lift by up to $25 \%$ as they sliced the laminar separation bubble into segments. Zaman, Bar-Severs \& Mangalam (1987) used acoustic excitation to control the flow over an LRN-(1)-1007 airfoil at Reynolds numbers of 40000-140 000, where small-amplitude excitation in a wide low-frequency range was found to eliminate laminar separation. Yarusevych, Sullivan \& Kawall (2005) indicated that the optimum acoustic excitation frequency should match that of the most amplified disturbance in the separated shear layer, which promoted the transition to bring earlier reattachment.

Studies of flow separation control have also been made for flow over a flat plate with adverse pressure gradient. Lang, Rist \& Wagner (2004) introduced a smallamplitude T-S wave and spanwise forcing of steady three-dimensional disturbances to control the transition development in a laminar separation bubble. Their experimental results showed that the transition was driven by a convective primary amplification of the two-dimensional T-S wave, while steady three-dimensional disturbances played a minor role. Marxen \& Rist (2010) studied the mutual interaction of laminar-to-turbulent transition and mean flow evolution in a separation bubble when a small disturbance was introduced upstream. The resultant mean flow change upstream of the transition location led to a reduction in the size of the separation regions as well as a stabilization of the flow. Rist \& Augustin (2006) and Postl, Balzer \& Fasel (2011) carried out flow separation control using instability waves and vortex generator jets, respectively, and they concluded that unsteady forcing was more effective than steady forcing in controlling laminar separation bubbles. When unsteady forcing was applied to the flow at the naturally unstable frequency of the separated shear layer, instability modes developed into large-scale spanwise coherent structures, initiating a necessary entrainment of high-momentum fluid to reattach the flow (Postl et al. 2011). A significant reduction in the size of the separation bubble was observed as a result of control.

\subsection{Plasma flow control}

Plasma flow control is a relatively new area of research, where the flow control is carried out by dielectric barrier discharge (DBD) plasma actuators. Typical plasma actuator consists of upper and lower electrodes that are attached to or embedded in a dielectric sheet. When the two electrodes are powered by a high-voltage, high-frequency AC power supply, the air over the upper electrode is ionized, creating a body force that acts on the ambient air. This creates a wall jet downstream of the plasma actuator (Jukes et al. 2006, 2008). Plasma actuators are all-electric devices without moving parts, which have many advantages over conventional actuators, including fast time response, simple construction and installation and the possibility of retrofitting.

An important aeronautical application of plasma actuators is airfoil flow control. Post \& Corke $(2004,2006)$ found that a DBD plasma actuator placed at the leading 
edge of an airfoil could control the static as well as dynamic stall. Sosa et al. (2007) placed a DBD plasma actuator over the suction side of an airfoil near the leading edge, where they found that the large-scale separation region was reduced or even eliminated, resulting in a large increment in lift. Mabe et al. (2009) used a DBD plasma actuator for flow separation control of a NACA 0021 airfoil. Due to the limited applicability of the device, the effectiveness of control was restricted to relatively low Reynolds number of 100000 , such as those associated with MAVs. Applying a DBD plasma actuator at the leading edge of a NACA 0015 airfoil at the Reynolds number of 260000 , Benard, Jolibois \& Moreau (2009) found that the stall angle was increased and the drag reduced. They found that an unsteady plasma actuation could result in a further increase in lift and a reduction in drag as compared to those by a steady actuation. Schuele \& Greenblatt (2010) applied a DBD plasma actuator near the leading edge of a thin airfoil with a Gurney flap at the Reynolds number of up to 20000 . The force measurement indicated that the plasma actuator increased the lift of an airfoil further.

Rizzetta \& Visbal (2011) carried out an LES to investigate the use of serpentineconfiguration DBD plasma actuators for flow control of an SD7003 airfoil at the Reynolds number of 40000 . Here, plasma actuators were placed near the leading edge of the airfoil to excite the transitional boundary layer by a pulsed mode at the frequency associated with the unstable shear layer. It was shown that the non-uniform spanwise forcing created by the plasma actuator suppressed the appearance of coherent two-dimensional modes to accelerate the transition process. Vorobiev, Rennie \& Jumper (2013) conducted flow separation control of a NACA 0009 airfoil at the Reynolds numbers of 70000-400000, where laser Doppler velocimetry (LDV) measurements were performed to study the effect of a DBD plasma actuator. The results indicated that the effectiveness of plasma actuators in trailing-edge separation control depended on the state of the boundary layer at the actuator location.

Plasma actuators have also been used for flapping airfoil control (Rizzetta \& Visbal 2012), airfoil roll control (Wei et al. 2013), closed-loop dynamic stall control (Lombardi, Bowles \& Corke 2013) as well as suppression of tonal trailing-edge noise from an airfoil (Inasawa, Ninomiya \& Asai 2013). Further details on the application of DBD plasma actuators for aerodynamic flow control can be found in recent review papers by Moreau (2007), Corke, Enloe \& Wilkinson (2010) and Wang et al. (2013).

\subsection{Gurney flaps}

The Gurney flap is a small boundary layer control device, which can be easily attached to the trailing edge of an airfoil (see figure 1). With only $2 \%$ of the chord of the airfoil, for example, the Gurney flap can increase the lift coefficient by 0.4 at the expense of a small drag. The lift-to-drag ratio can be enhanced by as much as $35 \%$ (Wang, Li \& Choi 2008). The mechanism of lift increase by the Gurney flap was studied and explained by Liebeck (1978). He showed that a pair of counter-rotating vortices was formed downstream of the Gurney flap (see figure 1), creating a lowpressure region within. This increased the velocity over the suction surface, giving rise to an increase in the suction pressure over the airfoil. At the same time, a recirculation region was created upstream of the Gurney flap, reducing the flow velocity there. As a result, the pressure on the pressure surface of the airfoil was increased. These changes in the pressure distribution led to an increase in the lift force on an airfoil with a Gurney flap. It was also thought that the vortex structure downstream of the Gurney flap extended the effective chord length and increased the effective camber of the airfoil. 


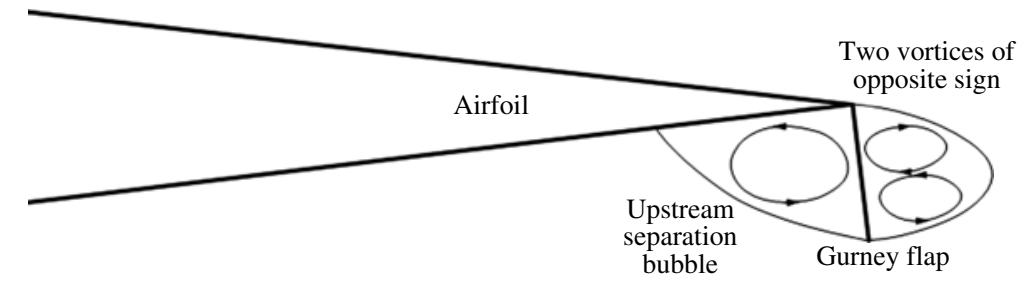

FIGURE 1. Flow modification by the Gurney flap at the trailing edge of an airfoil (Liebeck 1978).

Due to its simplicity, the Gurney flap has attracted much attention over the last four decades in both aeronautical and automotive applications. Li, Wang \& Zhang (2002, 2003) carried out a systematic investigation into the Gurney flap at the Reynolds number of 2100000 in order to find the optimum configuration. It was shown that the lift increased with an increase in the flap height when the Gurney flap was attached at the trailing edge normal to the airfoil. Li, Wang \& Hua (2007) and Yu, Wang \& Zhang (2011) found that the Gurney flap could improve the aerodynamic performance of supercritical airfoils at Reynolds numbers up to 5800000 .

Liu \& Montefort (2007) studied the lift on an airfoil with a Gurney flap using the thin-airfoil theory, showing that the lift and pitching moment increased proportional to the square root of the normalized flap height. Singh, Dhanalakshmi \& Chakrabartty (2007) performed a numerical simulation of NACA 0011 and 4412 airfoils with a Gurney flap at the Reynolds number of 2000000 , which agreed well with available experimental data. Kinzel, Maughmer \& Duque (2010) conducted a numerical investigation of oscillating airfoils with deployable Gurney flaps at the Reynolds number of 4000000 . It was shown that the chord-wise location of the Gurney flap greatly affected the unsteady aerodynamics of airfoils. However, the Gurney flap remained effective up to high deployment frequencies when it was located at the trailing edge.

A number of researchers have used the PIV technique to study the near-wake dynamics of an airfoil with a Gurney flap. Troolin, Longmire \& Lai (2006) found that two distinct vortex shedding modes interacted in the wake downstream of the airfoil. The dominant mode resembled Karman vortex shedding behind an asymmetric bluff body, while the second mode related to intermittent shedding of fluid recirculating in the cavity upstream of the Gurney flap. Lee and his coworkers (Lee 2009, 2011; Lee \& Ko 2009; Lee \& Su 2011) conducted a series of investigations on an airfoil with perforated Gurney flaps, where the near-wake characteristics associated with lift increment were analysed.

\subsection{Virtual Gurney flaps}

The Gurney flap is a useful high-lift device for aircraft, but it comes with a drag penalty at cruise since it cannot be stowed like other flaps. On the other hand, trailing-edge flaps of modern aircraft are often very large and heavy, and are operated by a mechanically complex system. In addition, the system components such as actuators and hinges to deploy and stow the flaps adversely affect the aerodynamics of aircraft. Addressing these issues, some novel active flow control methods have recently been proposed. For example, Traub, Miller \& Rediniotis (2004) developed a jet Gurney flap (see figure $2 a$ ), where a continuous jet was issued from a slot 
(a)

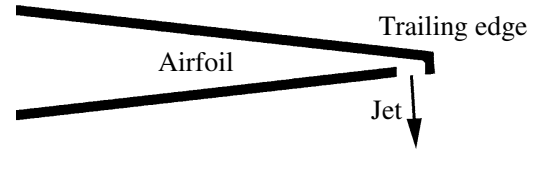

(c)

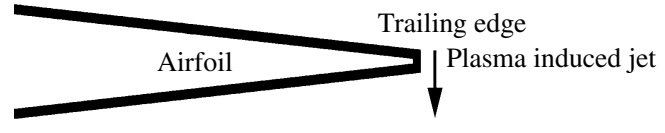

(e) (b)

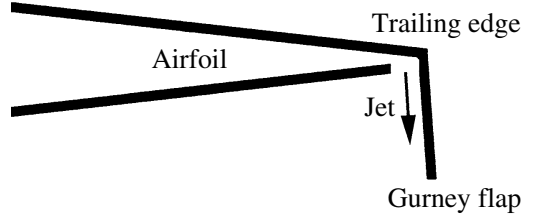

(d)

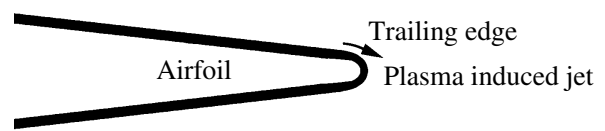

FIGURE 2. Sketch of the airfoil controlled by (a) jet (Traub et al. 2004), (b) Gurney flap with jet (Traub \& Agarwal 2008), (c) plasma induced jet (Zhang et al. 2009), (d) Gurney flap with plasma induced jet (Feng et al. 2012) placed near the sharp trailing edge, and by (e) plasma induced jet (Zhang et al. 2010) near the round trailing edge.

on the pressure surface of a NACA 0015 airfoil, $2 \%$ chord length upstream of the trailing edge. This resulted in lift and pitching moment increases, equivalent to a $0.75 \%$ chord Gurney flap with a jet momentum coefficient of $0.68 \%$. Traub \& Agarwal (2008) carried out an investigation of the Gurney flap in conjunction with a jet flap at low Reynolds numbers (see figure $2 b$ ), and found that the jet forcing could further increase the lift coefficient of the airfoil over that by the Gurney flap alone. Zhang, Liu \& Wang (2009), on the other hand, numerically simulated a DBD plasma actuator on the blunt trailing edge of a NACA 0012 airfoil (see figure 2c). Their results indicated that the downward wall jet from the plasma actuator increased the lift and nose-down pitching moment on the airfoil, similar to those of Gurney flaps. He, Corke \& Patel (2009) used a DBD plasma actuator as a jet flap by placing it near the trailing edge of a NACA 0015 airfoil. Little et al. (2010) and Little \& Samimy (2010) used a similar actuator at the flap shoulder of a simplified NASA EET airfoil for separation control. Circulation control was carried out by Rogers \& Donnelly (2004) by tangential jet injection near the trailing edge of an elliptic body, while Zhang et al. (2010) conducted a CFD study of an NCCR 1510-7067N airfoil with a DBD plasma actuator near the trailing edge (see figure $2 e$ ). Recently, Feng et al. (2012) placed a DBD plasma actuator over a Gurney flap attached to a NACA 0012 airfoil (see figure $2 d$ ), where the negative vertical velocity on the airfoil wake was increased by plasma control, leading to an increase in the lift coefficient.

In the present experimental investigation, we have carried out flow separation control using virtual Gurney flaps by simulating mechanical Gurney flaps with DBD plasma actuators. Here, a DBD plasma actuator was placed at the pressure side of a NACA 0012 airfoil near the trailing edge to direct the plasma-induced wall jet against the free stream. Velocity measurements around the airfoil model without and with plasma actuation were carried out using time-resolved PIV. At the same time, a force 


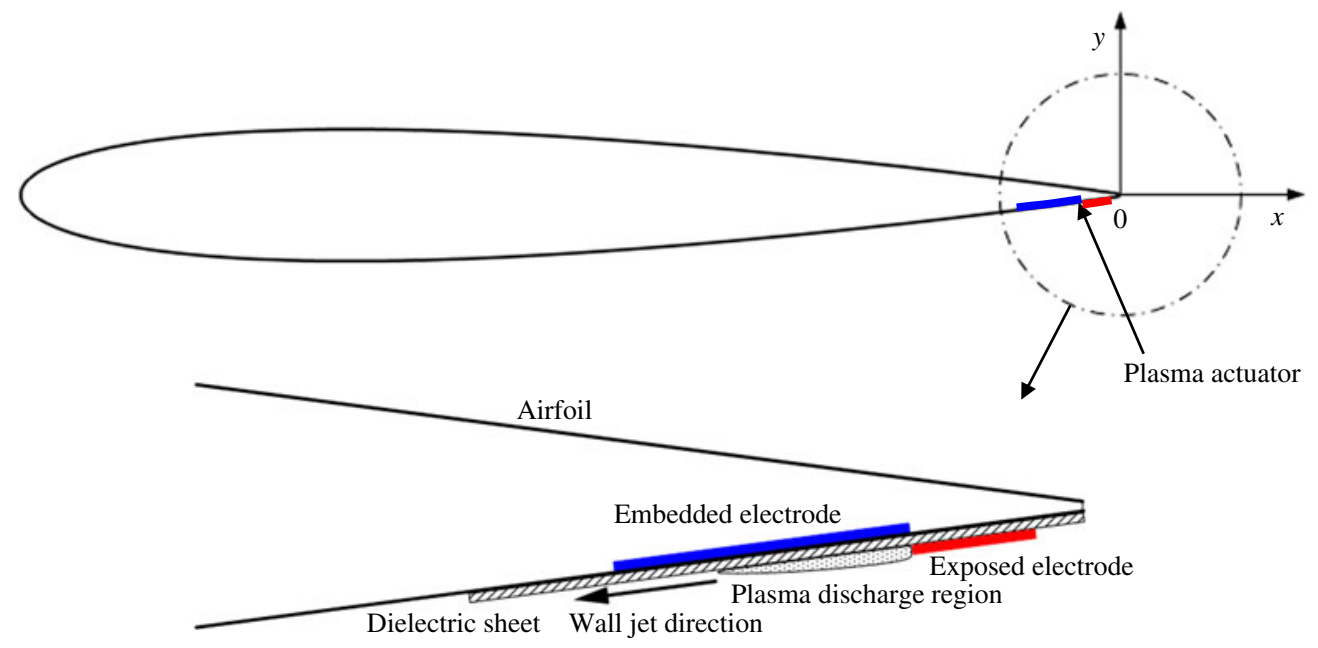

FIGURE 3. (Colour online) Sketch of NACA 0012 airfoil with DBD plasma actuator.

balance was also used to quantify lift and drag changes on the airfoil at different angles of attack. The behaviour of laminar separation bubbles over the airfoil was carefully monitored in conjunction with a development of the boundary layer, whose effect on the near-wake dynamics was clarified.

\section{Experimental setup}

Experiments were conducted in a low-speed, open-return wind tunnel with a $1.5 \mathrm{~m} \times 0.3 \mathrm{~m} \times 0.3 \mathrm{~m}$ test section. This was a suction tunnel, where the air was exhausted to the atmosphere. The turbulence level in the test section was $0.5 \%$ for the free-stream velocity of up to $5.5 \mathrm{~m} \mathrm{~s}^{-1}$. The experimental model used was a NACA 0012 airfoil with the chord length $c=100 \mathrm{~mm}$ and span $b=250 \mathrm{~mm}$, giving the aspect ratio $b / c=2.5$ (see figure 3 ). The airfoil was made of a foam material covered by a $100 \mu \mathrm{m}$ thick film to provide a smooth surface. End plates of $300 \mathrm{~mm} \times 200 \mathrm{~mm}$ in the streamwise and vertical directions, respectively, were mounted $25 \mathrm{~mm}$ from the wind tunnel walls to maintain the two-dimensionality of the flow field. Here, the origin of the coordinate system was fixed at the trailing edge of the airfoil, with the $x$ and $y$ axes pointing in the streamwise and vertical directions, respectively.

A DBD plasma actuator was placed on the pressure (lower) side of the airfoil near the trailing edge, as shown in figure 3. It consisted of a $2.5 \mathrm{~mm}$ wide exposed copper electrode and a $6 \mathrm{~mm}$ wide embedded copper electrode, which were separated by a $250 \mu \mathrm{m}$ thick Mylar dielectric sheet. The thickness of the copper electrodes was only $17 \mu \mathrm{m}$. The downstream edge of the exposed electrode was $1 \mathrm{~mm}$ upstream from the trailing edge of the dielectric sheet, whose upstream edge was extended by $3 \mathrm{~mm}$ beyond the end of the embedded electrode (see figure 3). This setup was necessary to avoid possible arcing from the exposed electrode to the embedded electrode on both sides. The DBD plasma actuator was powered sinusoidally at an AC voltage of $9.8 \mathrm{kV}_{p-p}$ with a frequency of $18 \mathrm{kHz}$, to produce a strong stable plasma body force. To compare results with the plasma actuator, a Gurney flap with $3.0 \mathrm{~mm}$ height (3\% chord) and $0.25 \mathrm{~mm}$ thick was also made, which can be attached at the training edge 
over the pressure (lower) side of the airfoil. This is similar in construction to that used in Feng et al. (2012).

We use the momentum coefficient of the plasma jet to quantify the momentum input of the plasma actuator. The momentum flux of a horizontal jet induced by the plasma actuator in quiescent air was calculated according to Jukes \& Choi (2009), who used time-resolved PIV measurements to estimate the plasma force based on the momentum theory. Here, an $18 \mathrm{~mm}$ wide control volume was chosen at $15 \mathrm{~mm}$ downstream of the exposed electrode edge, and the momentum flux $M$ was obtained by integrating the streamwise velocity. From this, the momentum coefficient of the plasma jet was calculated as $C_{\mu}=2 M / \rho U_{\infty}^{2} c$, where $\rho$ is the air density and $U_{\infty}$ is the free-stream velocity. In this investigation, the momentum flux of the plasma actuator was fixed, while the free-stream velocities were varied as $U_{\infty}=3.0,4.3$ and $5.3 \mathrm{~m} \mathrm{~s}^{-1}$, giving Reynolds numbers $R e=20000,28000$ and 35000 based on the airfoil chord length. This gave the corresponding momentum coefficients of the plasma jet $C_{\mu}=1.9 \%$, $0.9 \%$ and $0.6 \%$, respectively. Only results at the Reynolds number $R e=20000$ with $C_{\mu}=1.9 \%$ are presented in this paper. An exception is in figure 5, where the change in lift coefficient is demonstrated with an increase in the momentum coefficient.

The lift and drag coefficients were measured by a two-component force balance. The balance consisted of two parallelograms arranged in an ' $L$ ' shape. Each parallelogram was instrumented with four strain gauges, which were wired to form two Wheatstone bridges. The bridge input voltage was supplied by two Fylde FE-379-TA transducer amplifiers, while the bridge output was recorded at $2 \mathrm{kHz}$ using an IoTech 488/8SA analogue to digital converter and stored on a computer. More details on the force balance can be found in Jukes \& Choi (2009). The airfoil model was mounted into each force balance through a rod located at the middle axis of $25 \%$ chord length from the leading edge. The absolute accuracy of the force transducer was found to be better than $\pm 0.01 \mathrm{~N}$, corresponding to approximately $5 \%$ of the measured lift coefficient. The uncertainty of the airfoil attack angle in this investigation was within $\pm 0.25^{\circ}$.

The flow field was illuminated by a light sheet from a Litron LDY302-PIV $100 \mathrm{~W}$ Nd:YLF laser and the velocities were measured by a time-resolved PIV system from Dantec. The seeding particles were approximately $1 \mu \mathrm{m}$ diameter droplets generated from olive oil. The digital image sets were captured by a Phantom V12.1 high-speed camera, which had a spatial resolution of 1280 pixels $\times 800$ pixels in the streamwise and vertical directions, respectively. Two fields of view were measured around the airfoil for each test case. One had a range of $-1 \leqslant x / c \leqslant 0$ and $-0.1 \leqslant y / c \leqslant 0.4$, concerning the evolution of the separation bubble over the suction surface, while the other had a range of $-0.3 \leqslant x / c \leqslant 0.4$ and $-0.25 \leqslant y / c \leqslant 0.25$, concerning the wake vortex dynamics near the trailing edge. The sampling frequency was $2 \mathrm{kHz}$. For each test case, 4000 image pairs were recorded continuously, with the first 2000 image pairs without plasma control and the rest with plasma control. The Dantec Dynamic Studio v3.00 was used to calculate the velocity and vorticity fields. For the first field of view, the interrogation window was set to 16 pixel $\times 16$ pixel with $50 \%$ overlap in both streamwise and vertical directions, while it was 32 pixel $\times 32$ pixel with $50 \%$ overlap for the second one. A recursive cross-correlation algorithm was used to compute the velocity vector maps. The spatial resolution of the velocity vectors was approximately $1 \mathrm{~mm}$, corresponding to approximately $0.01 c$, which is sufficient to capture the separation bubble and the vortical structures. 

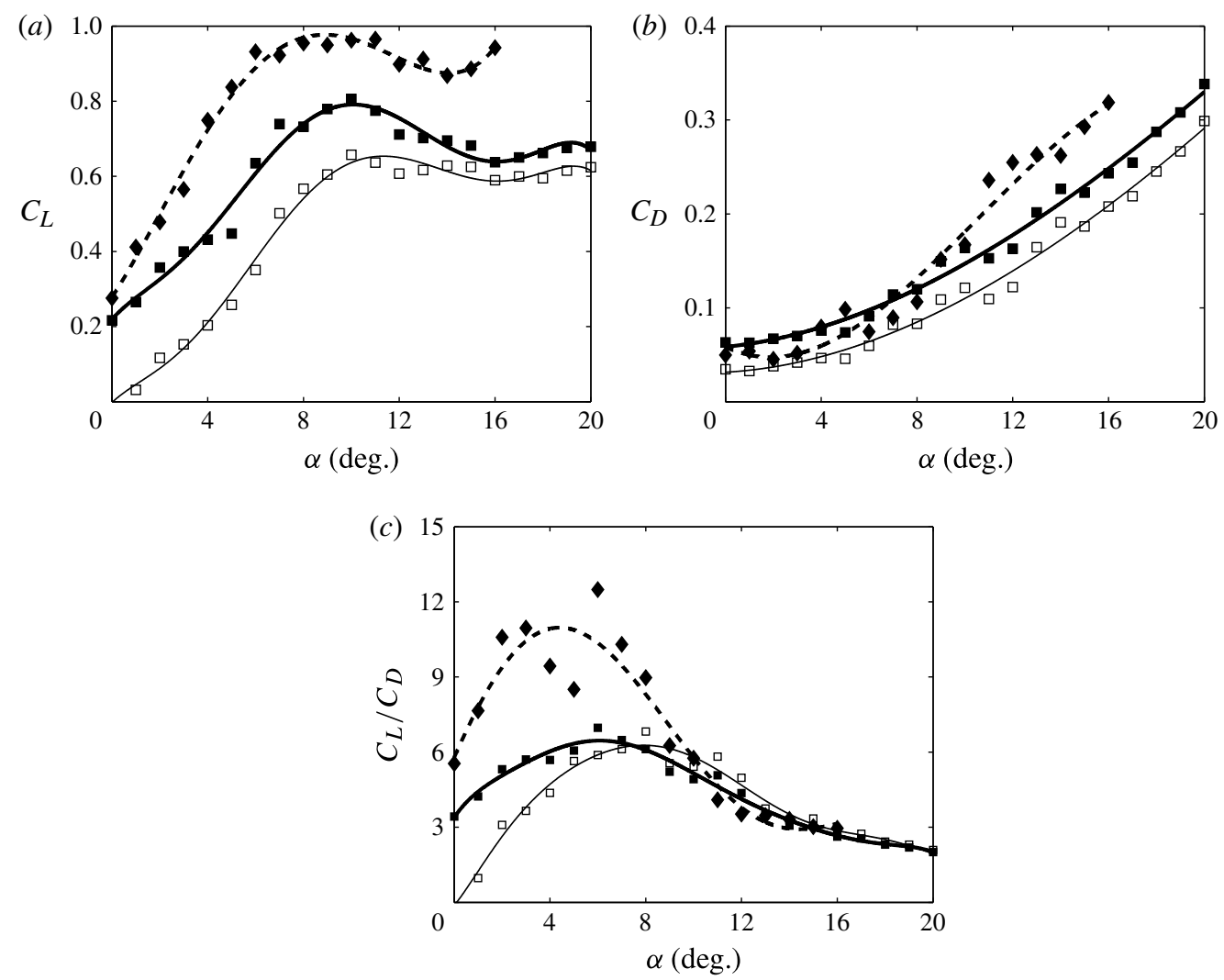

FIGURE 4. (a) Lift coefficient, $(b)$ drag coefficient and (c) lift-to-drag ratio, versus angle of attack at $R e=20000: \square$, plasma off; $\mathbf{n}$, plasma on with $C_{\mu}=1.9 \% ; \diamond, 3 \% c$ mechanical Gurney flap. The thin solid, thick solid, and thick dashed lines denote the fitting curves of without plasma, with plasma, and with Gurney flap, respectively.

\section{Lift and drag forces}

Figure 4 shows the aerodynamic force coefficients $C_{L}, C_{D}$ and the lift-to-drag ratio $C_{L} / C_{D}$ as a function of the angle of attack $\alpha$ at $R e=20000$. Figure 4(a) indicates that the lift slope is not constant even for small angles of attack due to low Reynolds numbers (Kim et al. 2011). It also shows that plasma control with jet momentum coefficient of $C_{\mu}=1.9 \%$ increases $C_{L}$ for all angles of attack tested here, producing a maximum lift enhancement of $23 \%$. It also increases the drag coefficient $C_{D}$ for all angles of attack as shown in figure $4(b)$. As a result, the lift-to-drag ratio is increased by plasma control until the stall angle, as shown in figure $4(c)$. However, there seems to be no increase in $C_{L} / C_{D}$ at post-stall angles of attack. The aerodynamic characteristics of the airfoil with a $3 \%$ chord Gurney flap is also given in figure 4 for comparison, showing similar characteristics to that of the virtual Gurney flap. However, increases in $C_{L}, C_{D}$ and $C_{L} / C_{D}$ by a $3 \%$ chord Gurney flap are nearly always greater than those by plasma control with $C_{\mu}=1.9 \%$, although the drag penalty of the mechanical Gurney flap is slightly less than that of the virtual Gurney flap for $\alpha<8^{\circ}$. This will be explained below.

Figure 5 shows the increase in lift coefficient, $\Delta C_{L}$ by plasma control at zero angle of attack as a function of the plasma jet momentum coefficient, $C_{\mu}$. This 


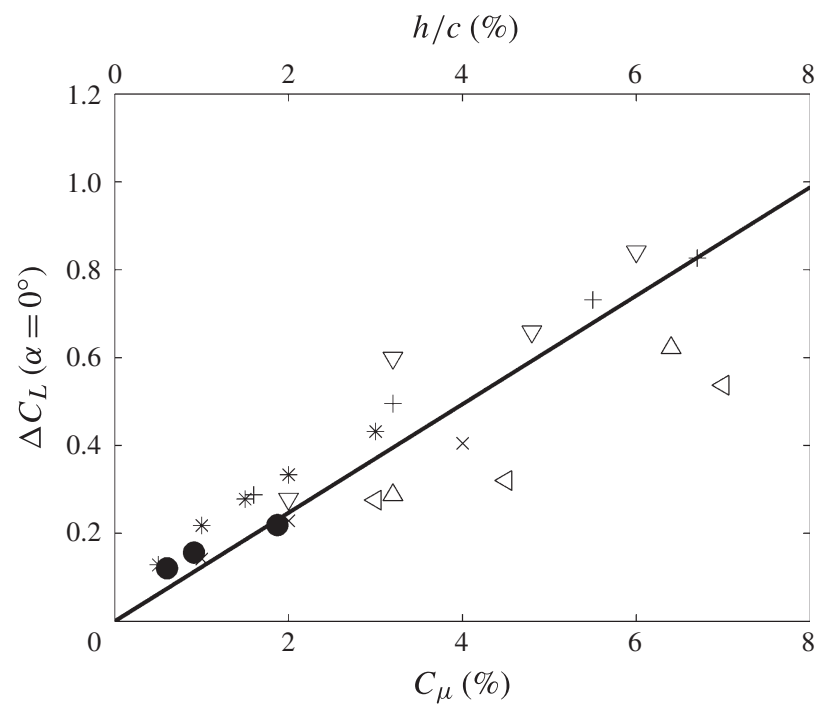

FIGURE 5. Increase in lift coefficient by plasma forcing at zero angle of attack as a function of moment coefficient $C_{\mu}$. The corresponding value by mechanical Gurney flap from related references is given as a function of height $h / c . \triangle$, Lee \& Ko (2009), $R e=$ $53000 ; \nabla$, Lee (2011), $R e=105000 ;+$, Lee (2009), $R e=232000 ; \times$, Jeffrey \& Hurst (1996), $R e=850000 ; *$, Li et al. (2002), $R e=2100000 ; \boldsymbol{\bullet}$, virtual Gurney flap with plasma forcing at $C_{\mu}=0.6 \%(R e=35000), 0.9 \%(R e=28000)$, and $1.9 \%(\operatorname{Re}=20000)$; $\triangleleft$, present Gurney flap, $R e=20000$; the straight line represents the least-square fit through the data.

result suggests that $\Delta C_{L}$ increases nearly linearly with the jet momentum coefficient $C_{\mu}$ of plasma control, giving $\Delta C_{L} \approx 12 C_{\mu}$. A similar behaviour is observed in the Gurney flap data over a wide range of the Reynolds number, as shown in figure 5, indicating that $\Delta C_{L}$ increases linearly with the height $h / c$ of the Gurney flap, giving $\Delta C_{L} \approx 12 h / c$. Figure 5 indicates that the lift enhancement by plasma control with a $1 \%$ momentum coefficient is equivalent to a $1 \%$ chord Gurney flap. A similar finding was made by Traub et al. (2004), who showed that the jet Gurney flap with $C_{\mu}=0.68 \%$ gave a lift enhancement equivalent to a Gurney flap of $h / c=0.75 \%$.

\section{Flow over an airfoil}

\subsection{Time-averaged characteristics}

Figure 6 shows time-averaged streamlines for an airfoil without and with plasma control at different angles of attack. Without DBD plasma actuation, flow over the suction (upper) surface is fully attached at small angles of attack up to $\alpha=2^{\circ}$ (figure $6 a, c$ ), while it starts to separate near the trailing edge at $\alpha=4^{\circ}$, forming a small separation region there (figure $6 e$ ). At these angles of attack, streamlines are not changed much by plasma control (figure $6 b, d, f$ ). As the angle of attack increases to $\alpha=6^{\circ}$, the separation point moves upstream with the appearance of a clear separation bubble near the trailing edge (figure $6 g$ ). Plasma control makes the bubble become smaller (figure 6h), while the location moves further upstream. This reduction in size of the laminar separation bubble is reflected in the shift of peak lift-to-drag ratio to $\alpha=6^{\circ}$ in figure $4(c)$. As the angle of attack increases to $\alpha=8^{\circ}$ 


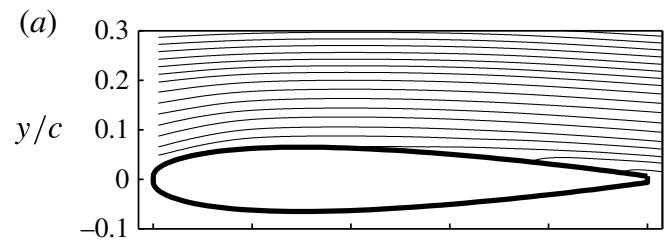

(b)

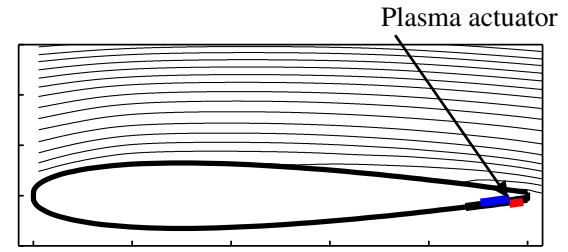

(c)

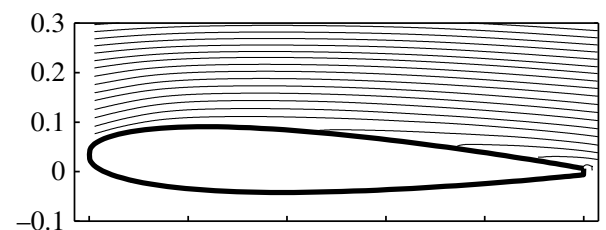

(d)

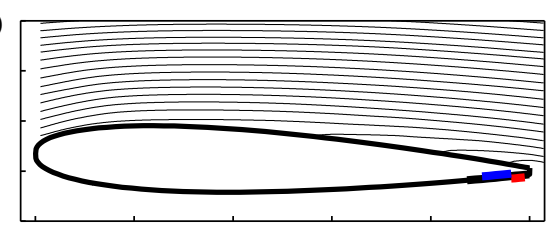

(e)

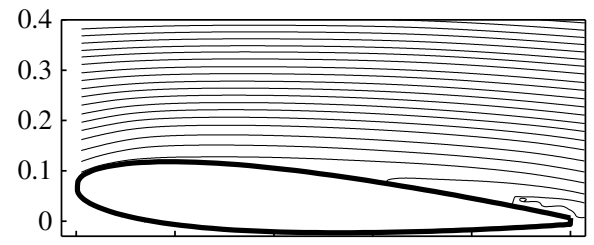

$(f)$

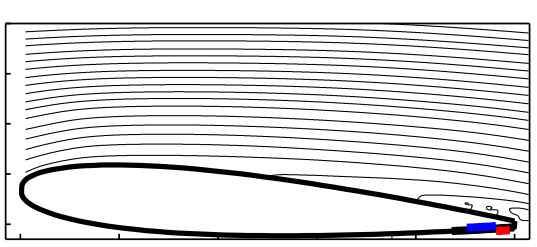

(g)

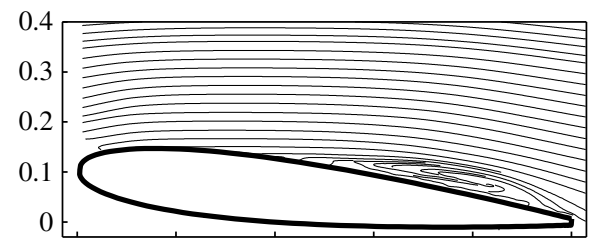

(h)

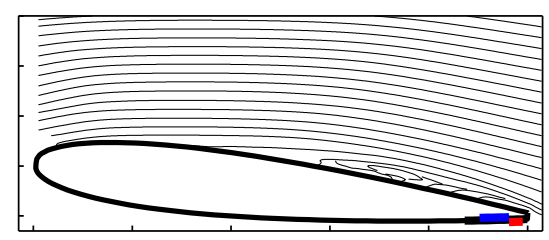

(i)

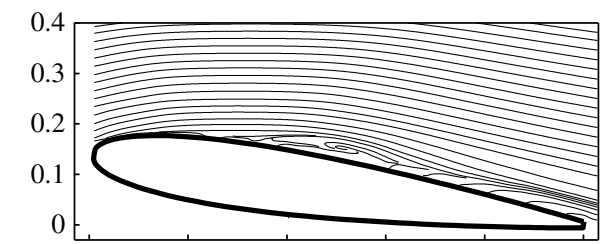

$(j)$

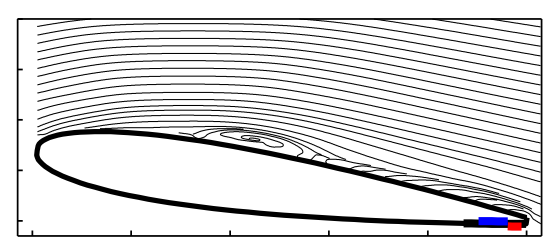

(k)

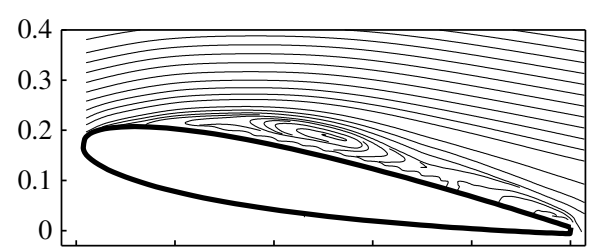

$(l)$
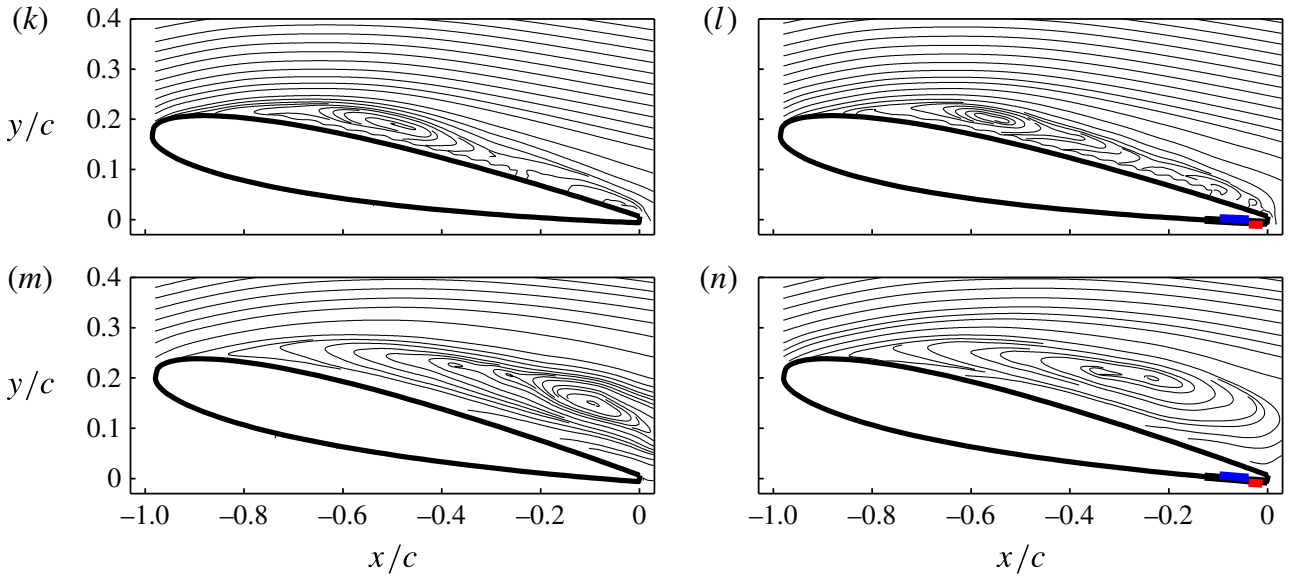

(n)

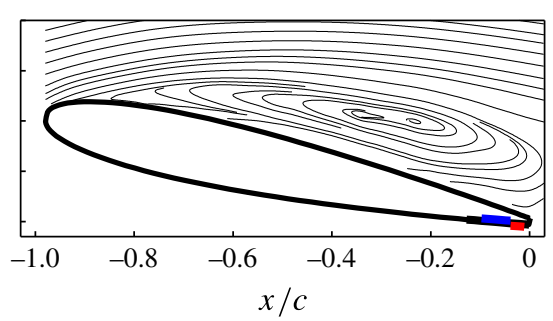

FIgURE 6. (Colour online) Time-averaged streamlines over an airfoil without $(a, c, e, g, i, k, m)$ and with $(b, d, f, h, j, l, n)$ plasma control at $R e=20000, C_{\mu}=1.9 \%$. $(a, b)$ $\alpha=0^{\circ} ;(c, d) \alpha=2^{\circ} ;(e, f) \alpha=4^{\circ} ;(g, h) \alpha=6^{\circ} ;(i, j) \alpha=8^{\circ} ;(k, l) \alpha=10^{\circ} ;(m, n) \alpha=12^{\circ}$. 

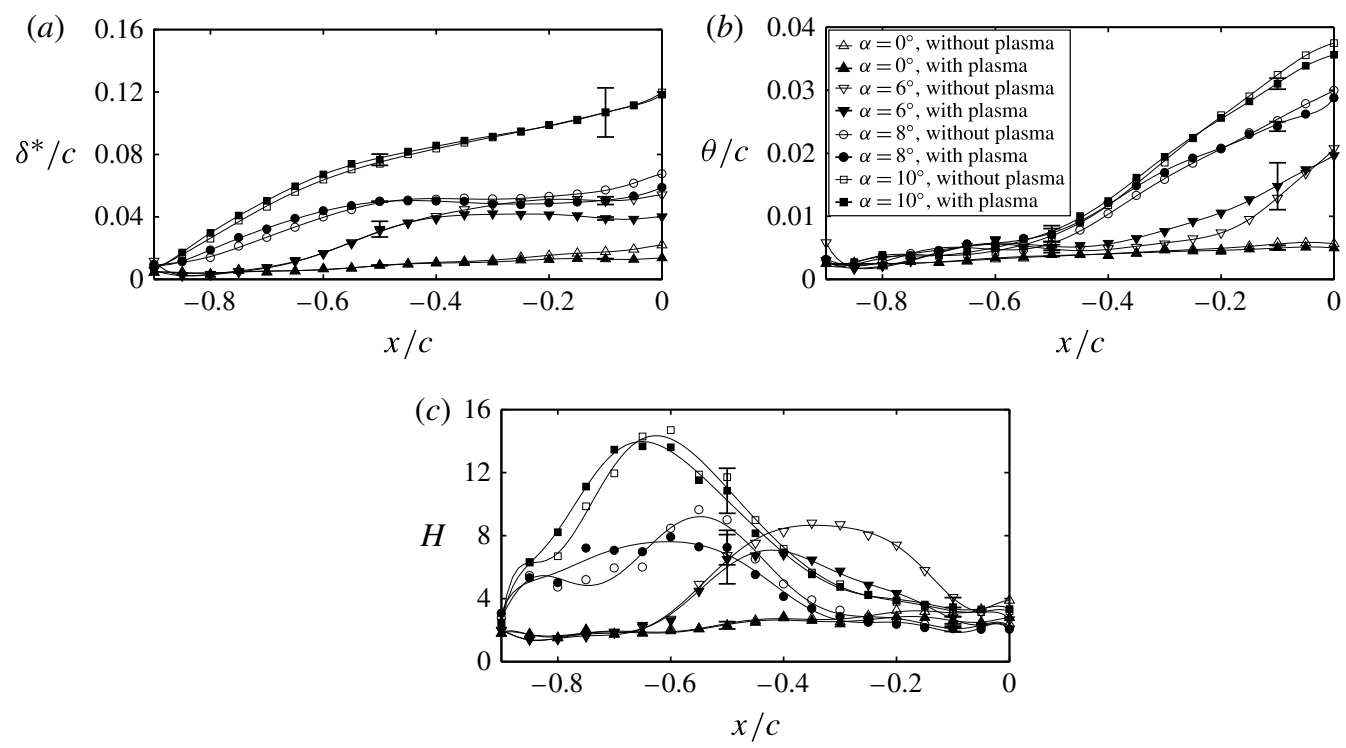

FIGURE 7. Boundary layer integral parameters over the airfoil suction (upper) surface without and with plasma control at $R e=20000$ and $C_{\mu}=1.9 \%$. (a) Displacement thickness $\delta^{*} ;(b)$ momentum thickness $\theta ;(c)$ shape factor $H$. The empty and filled symbols denote cases without and with plasma control, respectively. The error bars relate to the plasma control cases.

and $10^{\circ}$, the separation bubble moves further upstream (figure $6 i, k$ ), although there is little difference in the bubble size with plasma control (figure $6 j, l$ ). The flow over the airfoil is fully separated at $\alpha=12^{\circ}$ (figure $6 \mathrm{~m}$ ), exhibiting a large-scale recirculation region. Figure $6(n)$ shows that plasma control cannot affect the region that is already separated at this angle of attack.

Figure 7 shows changes in integral boundary layer parameters over the airfoil suction surface. Without control, both the boundary layer displacement thickness $\delta^{*}$ and the momentum thickness $\theta$ increase monotonically with $x / c$, while the shape factor $H$ exhibits a peak that moves upstream with an increase in the angle of attack. It should be noted that the reverse flow within a laminar separation bubble increases the displacement thickness $\delta^{*}$, while the momentum thickness $\theta$ is reduced by it. Therefore, the shape factor $H=\delta^{*} / \theta$ of the boundary layer increases when a laminar separation bubble is contained within. In other words, the peak in the shape factor $H$ can be considered as the location of the centre of a laminar separation bubble. The plasma control increases the displacement thickness in the upstream region of the airfoil $(x / c<-0.5)$ at $\alpha=8^{\circ}$ and $10^{\circ}$, while $\delta^{*}$ is reduced downstream $(x / c>-0.5)$ at the angle of attack of $\alpha=0^{\circ}-8^{\circ}$, as shown in figure $7(a)$. On the other hand, figure $7(b)$ indicates that the momentum thickness is increased with plasma control in the mid-chord region at the angle of attack of $\alpha=6^{\circ}-10^{\circ}$. As a result, the shape factor $H$ is reduced by the virtual Gurney flap at the angle of attack of $\alpha=6^{\circ}$ in the downstream region where the laminar separation bubble is located (figure $7 c$ ). At $\alpha=8^{\circ}$ and $10^{\circ}$, plasma control increases the shape factor $H$ in the front (upstream) part of the laminar separation bubble, while $H$ is reduced to the rear (downstream). 

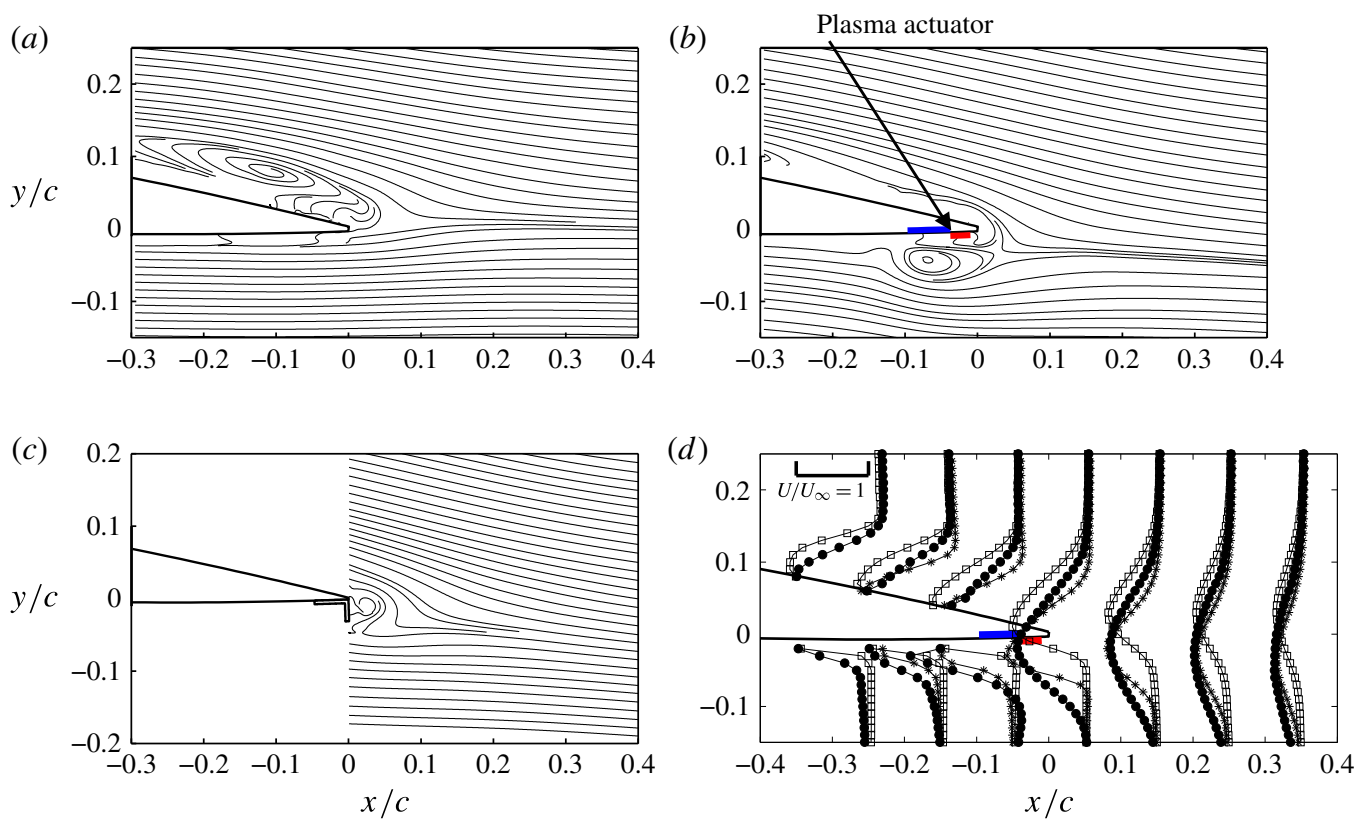

FIGURE 8. (Colour online) Flow field around the airfoil at $\alpha=6^{\circ}$ with $R e=20000$ and $C_{\mu}=1.9 \%$. (a) Time-averaged streamlines without plasma control; $(b)$ time-averaged streamlines with plasma control; $(c)$ time-averaged streamlines with $3 \% c$ mechanical Gurney flap; $(d)$ comparison of time-averaged streamwise velocity: - $\square-$, without plasma control; -๑-, with plasma control; -*-, $3 \% c$ mechanical Gurney flap.

\subsection{Dynamics of near-wake flow}

Figure 8 shows the time-averaged flow field near the trailing edge of the airfoil without and with plasma control at an angle of attack $\alpha=6^{\circ}$. A separation bubble is clearly seen over the suction (upper) surface near the trailing edge (figure $8 a$ ). When the plasma actuator is activated (figure $8 b$ ), the plasma wall jet interacts with the free stream to form a recirculation region over the pressure (lower) surface of the airfoil. The recirculation region stretches downstream beyond the trailing edge, effectively extending the airfoil chord length. This is similar to the flow field created by the mechanical Gurney flap (see figures 1 and $8 c$ ), although only a single recirculation region is seen with plasma control as compared to two separate recirculation regions (one upstream of the flap and other downstream of it) with the Gurney flap. It draws the air from the suction (upper) surface, shifting the near wake downwards (figure $8 b$ ). Figure $8(d)$ shows that the formation of a recirculation region by the plasma actuator reduces the velocity over the pressure (lower) surface, while the velocity over the suction (upper) surface is increased, similar with that induced by a mechanical Gurney flap. As a result, the flow separation over the upper surface near the trailing edge (figure $8 a$ ) has been eliminated by the plasma actuator (figure $8 b$ ). Therefore, the lift enhancement by the virtual Gurney flap shown in figure 4 can also be considered as a result of the pressure difference across the airfoil created by the recirculation region. The near-wake flow fields at other angles of attack have also been studied to show that the recirculation region always plays an important role in lift enhancement, although its size decreases with an increase in the angle of attack. 
(a)

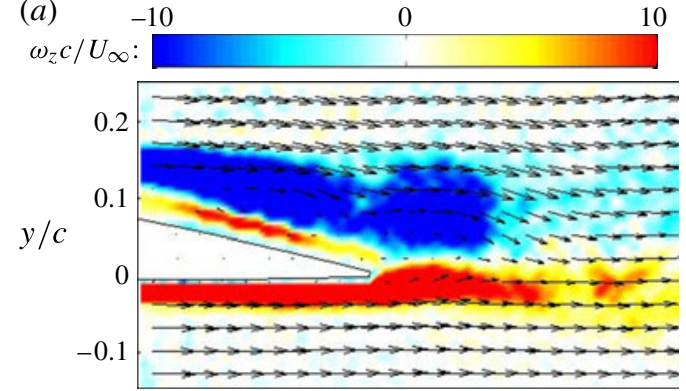

(c)

$y / c$
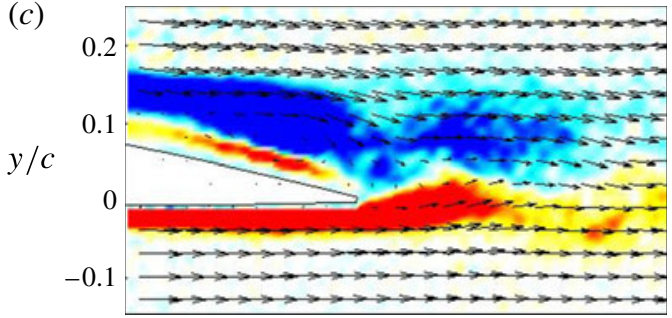

(e) 0.2

$y / c$

0.1
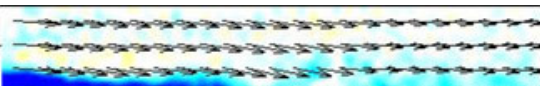

0

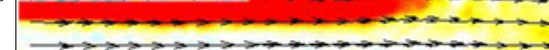

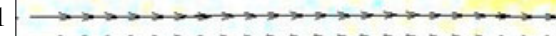

(g)

$y / c$

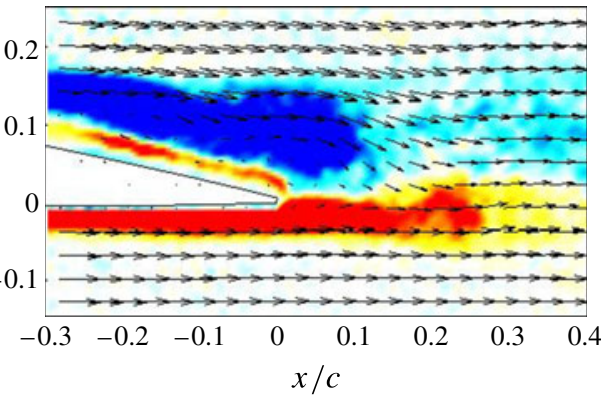

(b) $V / U_{\infty}$ :

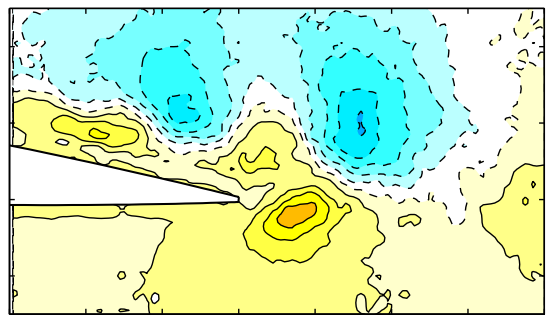

(d)

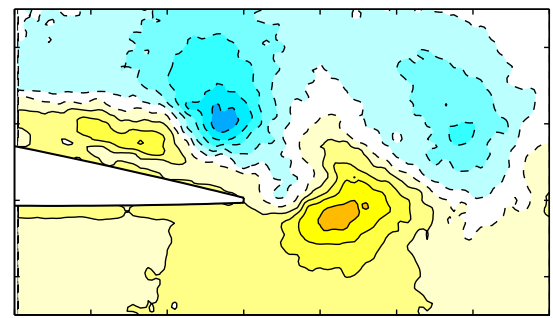

$(f)$

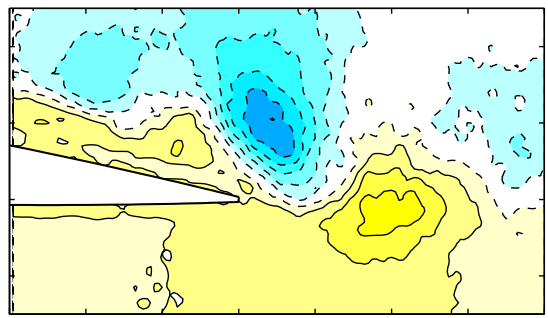

$(h)$

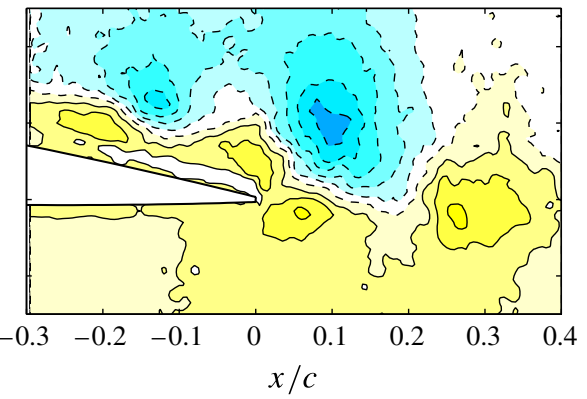

FIGURE 9. (Colour online) Evolution of phase-averaged spanwise vorticity $\omega_{z} c / U_{\infty}$ $(a, c, e, g)$ and vertical velocity $V / U_{\infty}(b, d, f, h)$ at four phases: $(a, b) \Phi=0^{\circ},(c, d) \Phi=90^{\circ}$, $(e, f) \Phi=180^{\circ},(g, h) \Phi=270^{\circ}$, for the airfoil without plasma control at $\alpha=6^{\circ}, R e=$ 20000 .

The modification by the plasma actuator of the near-wake topology suggests a variation in the near-wake dynamics, which is presented in figures 9 and 10 by the phase-averaged flow field at an angle of attack $\alpha=6^{\circ}$. The phase averaging was carried out with reference to the streamwise velocity at $x / c=0.10, y / c=0.16$, following the methodology in Feng \& Wang (2010). Here, the phase angle of $\Phi=0^{\circ}$ corresponds to the maximum velocity. The phase-averaged spanwise vorticity without plasma is shown in figure $9(a, c, e, g)$, while the corresponding phase-averaged vertical 
(a)

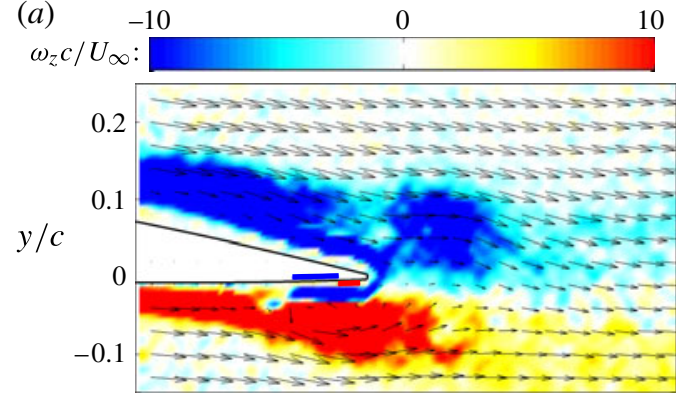

(c)

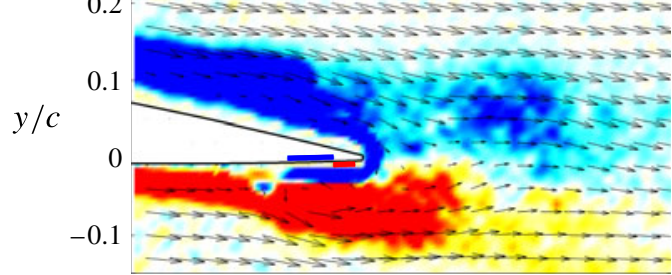

(e)

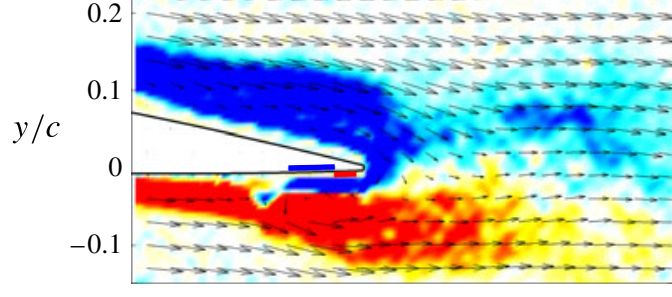

$(g)$

$$
y / c^{0}
$$

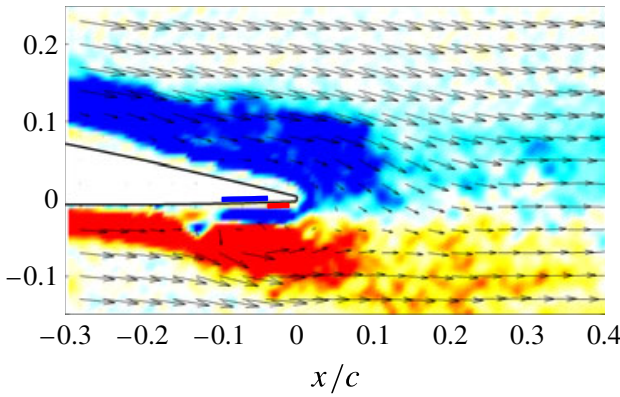

(b)

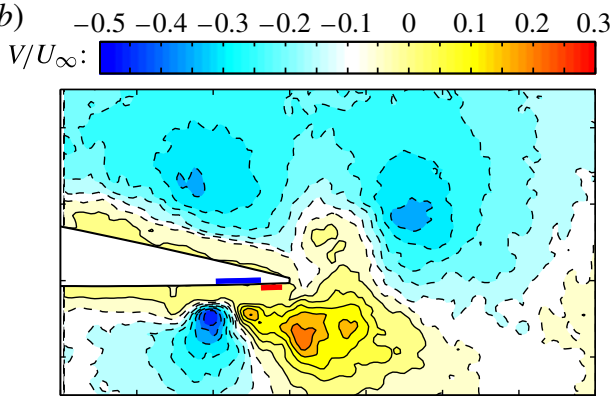

(d)

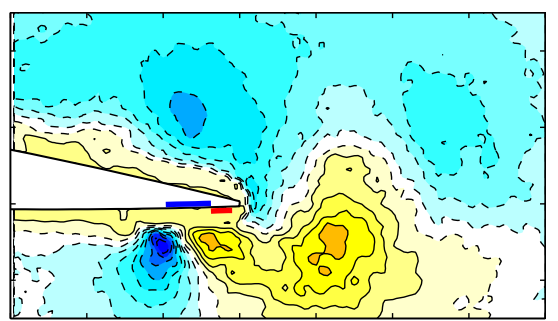

$(f)$

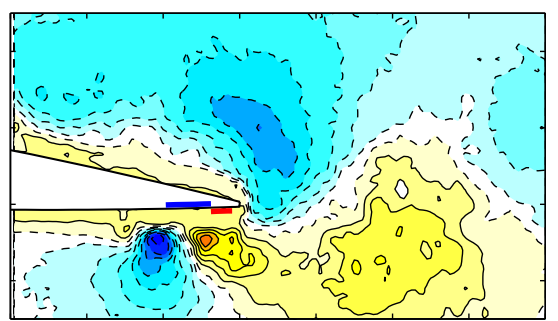

(h)

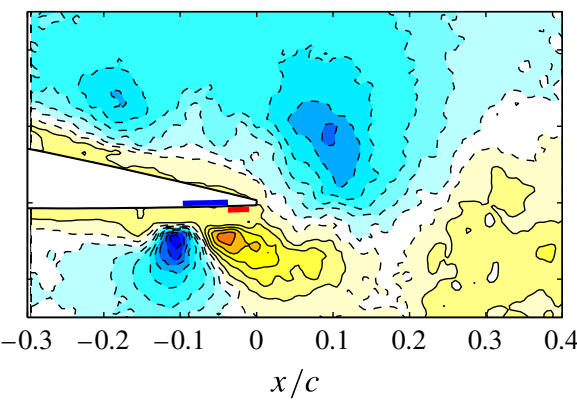

FIGURE 10. (Colour online) Evolution of phase-averaged spanwise vorticity $\omega_{z} c / U_{\infty}$ $(a, c, e, g)$ and vertical velocity $V / U_{\infty}(b, d, f, h)$ at four phases: $(a, b) \Phi=0^{\circ},(c, d) \Phi=90^{\circ}$, $(e, f) \Phi=180^{\circ},(g, h) \Phi=270^{\circ}$, for the airfoil with plasma control at $\alpha=6^{\circ}, R e=20000$, $C_{\mu}=1.9 \%$.

velocity is shown in figure $9(b, d, f, h)$. Here, a reverse flow due to flow separation is visible on the suction (upper) surface, creating a positive shear layer close to the surface near the trailing edge. It also shows a shedding of negative vortices from just upstream of the airfoil trailing edge (at around $x / c=-0.1$ ), drawing the positive vorticity from within the flow separation region to form a Karman vortex street.

With plasma control, a quasi-steady recirculation region appears near the plasma actuator over the pressure (lower) side of the airfoil near the trailing edge 
(figure 10a,c,e,g). This draws the air from the suction (upper) surface, nearly eliminating the trailing-edge flow separation. Here, the reattachment of separated flow with plasma control at $\alpha=6^{\circ}$ can be confirmed by the disappearance of the positive vorticity layer (figure $10 a, c, e, g$ ) and the vanishing positive vertical velocity (figure $10 b, d, f, h$ ) over the suction (upper) surface. The vortex shedding seems to start only downstream of the trailing edge. Figure 10 also shows that the quasi-steady recirculation region disperses the positive shear layer coming off the trailing edge. The recirculation region formed by the plasma control also shows the quasi-steady characteristics at other angles of attack (not shown here).

\subsection{Flow separation and reattachment}

Figure 11 shows the root-mean-squared (r.m.s.) velocity profiles over the airfoil suction surface without and with plasma control. It is found that the streamwise r.m.s. velocity starts to grow at or just downstream of the leading edge of the airfoil at $\alpha=6^{\circ}-10^{\circ}$. Such distributions are similar to those in previous investigations into the flow over a flat plate (Kiya \& Sasaki 1983) and over an airfoil (Boutilier \& Yarusevych 2012; Wahidi \& Bridges 2012) at low Reynolds numbers. It shows that the local peak value of streamwise r.m.s. velocity is much higher than that of vertical r.m.s. velocity at the start, indicating an initial development of T-S waves over the airfoil. This is followed by the vertical r.m.s. velocity increase to a value comparable to that of the streamwise r.m.s. velocity, indicating a subsequent growth of $\mathrm{K}-\mathrm{H}$ instability. The vertical position of their peaks, which is very close to the local boundary layer displacement thickness, moves away from the airfoil surface gradually in the downstream direction. With plasma control, the magnitude of r.m.s. velocities is similar to that of the natural case except for the near-wall region, although their profiles are shifted closer to the airfoil surface at $\alpha=6^{\circ}$.

The behaviour of laminar boundary layer transition during flow separation and subsequent reattachment over the airfoil has been analysed based on the PIV data. Here, the start and end of transition were determined as the initiation and completion of exponential growth in the maximum Reynolds shear stress across the boundary layer (Burgmann \& Schröder 2008). Figure 12(a) shows the logarithm of the maximum Reynolds shear stresses $\left(-\overline{u v} / U_{\infty}^{2}\right)_{\max }$ as a function of $x / c$ over the airfoil at $\alpha=6^{\circ}, 8^{\circ}$ and $10^{\circ}$, where straight lines were fitted using the least-square method. The start and end points of straight lines through the exponential growth of the maximum Reynolds shear stress were adjusted to maximize the correlation coefficient of the line fit, which was greater than 0.97 in all cases presented here. With an increase in the angle of attack, it is observed that the start and end of transition are moved upstream while the growth rate of the maximum Reynolds shear stress is increased. With plasma control at angles of attack of $\alpha=6^{\circ}$ and $10^{\circ}$, the growth rate of the maximum Reynolds stress is reduced, while it is increased at $\alpha=8^{\circ}$. The distributions of the maximum streamwise and vertical r.m.s. velocities are also plotted in figure $12(b, c)$ for comparison. The behaviour of the exponential growth for $\left(v^{\prime} / U_{\infty}\right)_{\max }$ is similar with that for $\left(-\overline{u v} / U_{\infty}^{2}\right)_{\max }$, suggesting that the boundary layer transition over the airfoil at this Reynolds number is dominated by K-H instability (Hatman \& Wang 1999).

The flow separation point was obtained based on particle traces obtained by the PIV measurement, while the reattachment point was determined by analysing streamlines over the airfoil (Burgmann et al. 2008). The centre of the laminar separation bubble was obtained by detecting the nodal point surrounded by spiral streamlines inside the 

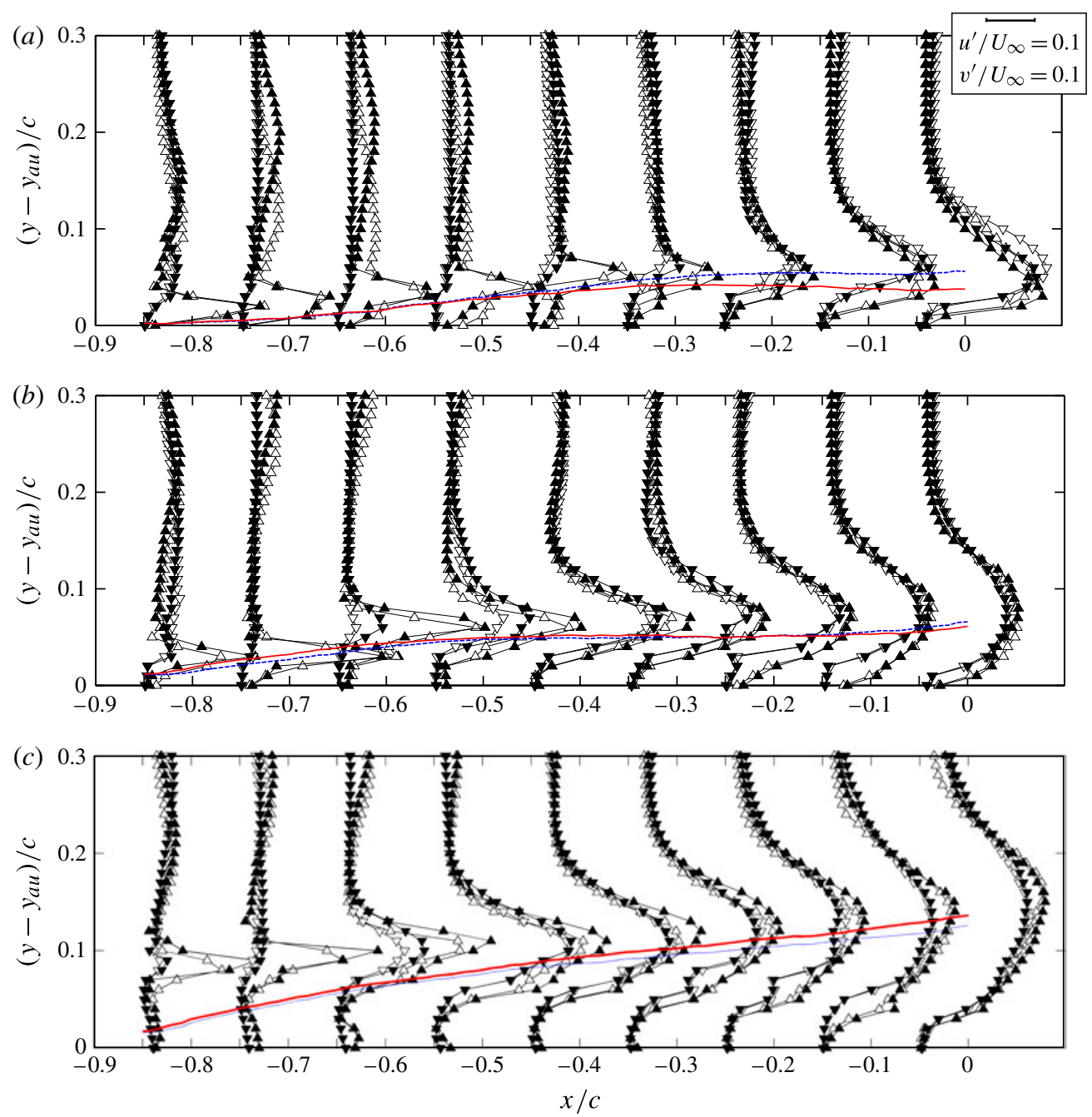

FIGURE 11. (Colour online) Root-mean-squared (r.m.s.) velocity profiles over the airfoil suction (upper) surface without and with plasma control at $(a) \alpha=6^{\circ}$, , b ) $8^{\circ}$, (c) $10^{\circ}$, and $R e=20000, C_{\mu}=1.9 \%:-\triangle-, u^{\prime} / U_{\infty}$ without control, $-\mathbf{\Delta}-, u^{\prime} / U_{\infty}$ with control; $-\nabla-, v^{\prime} / U_{\infty}$ without control, $-\nabla-, v^{\prime} / U_{\infty}$ with control. The streamwise positions of the profiles are from $x / c=-0.85$ to -0.05 with an interval of 0.1 , and $y_{a u}$ stands for the vertical distance from the upper surface of the airfoil. The dashed and solid lines denote the displacement thickness for the airfoil without and with plasma control, respectively.

bubble. These results are shown in figure 13 for the angle of attack of $\alpha=4^{\circ}-10^{\circ}$. Only the separation point and the bubble centre are shown for $\alpha=4^{\circ}$, since the other parameters were not obtainable at this angle of attack. As the angle of attack increases, the start and end of transition, the centre of the laminar separation bubble and the separation point move upstream, as we expected from the result shown in figure 6. The upstream movement of the laminar separation bubble with an increase in the angle of attack over low-Reynolds-number airfoils has also been observed in other investigations (Burgmann et al. 2008; Yang \& Hu 2008; Hain et al. 2009; 

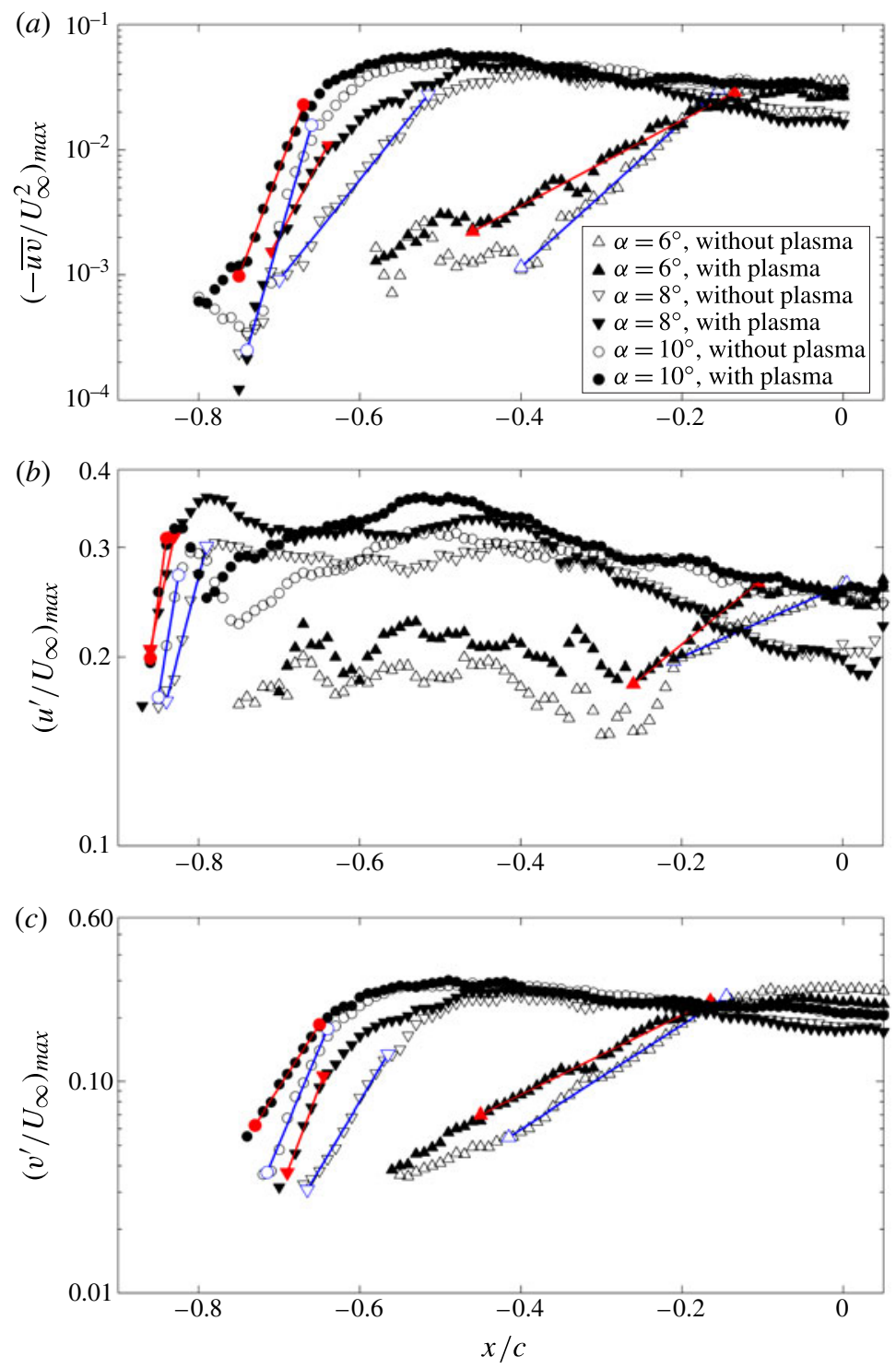

FIgURE 12. (Colour online) Distributions of $(a)$ the maximum Reynolds shear stress $\left(-\overline{u v} / U_{\infty}^{2}\right)_{\max }$, (b) the maximum streamwise r.m.s. velocity $\left(u^{\prime} / U_{\infty}\right)_{\max }$, and $(c)$ the maximum vertical r.m.s. velocity $\left(v^{\prime} / U_{\infty}\right)_{\max }$ over the airfoil suction (upper) surface without and with plasma control at $R e=20000$ and $C_{\mu}=1.9 \%$. Straight lines denote the exponential growth of the maximum value without and with plasma control, respectively, where the start and the end of the exponential growth are marked.

Boutilier \& Yarusevych 2012). Our result shows that the laminar-to-turbulent transition starts downstream of the flow separation point, but before the centre of the laminar separation bubble. The end of transition is located just upstream of the centre of the separation bubble at $\alpha=8^{\circ}$ and $10^{\circ}$, although it occurs further downstream close to the reattachment point at $\alpha=6^{\circ}$. 


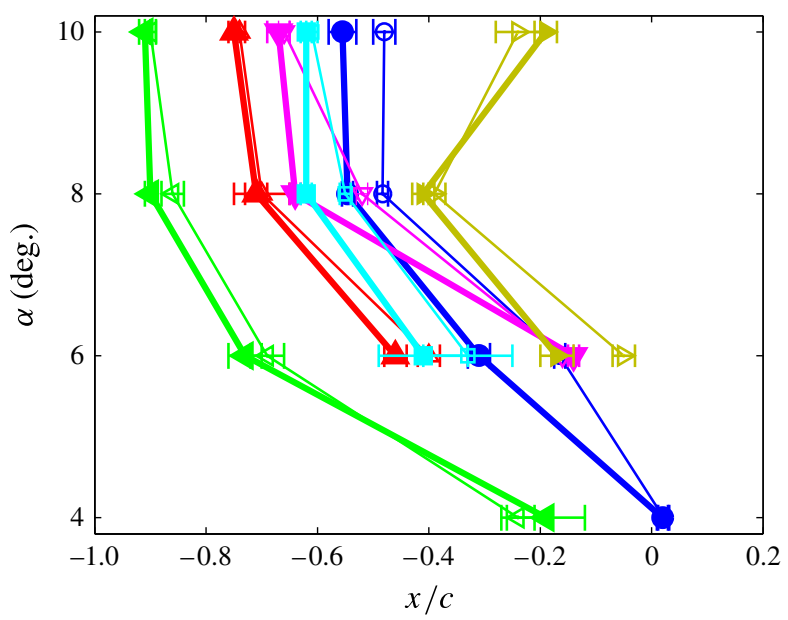

FIgURE 13. (Colour online) Statistical parameters of the separation bubble over the airfoil suction (upper) surface without and with plasma control at $R e=20000$ and $C_{\mu}=1.9 \%$ :

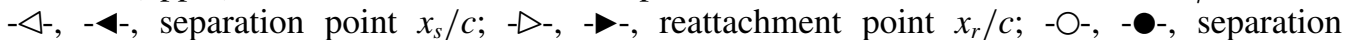
bubble centre $x_{c} / c ;-\triangle-,-\mathbf{\Delta}-$, start of transition $x_{s t} / c ;-\nabla-,-\nabla-$, end of transition $x_{e t} / c ;-\square-$, - - -, point of peak shape factor. The open symbols with thin lines and the filled symbols with thick lines denote cases without and with plasma control, respectively.

With plasma control, the flow separation point as well as the start and end of transition are advanced for the angle of attack of $\alpha=6^{\circ}-10^{\circ}$. The centre of the laminar separation bubble is also shifted upstream with control. An upstream shift of the reattachment point is also evident in the results, but only at $\alpha=6^{\circ}$. These behaviours are in line with the development of the shape factor $H$, as shown from the variations of the point of peak shape factor. Conducting a wind tunnel test of a NACA 0012 airfoil at the Reynolds number of 2100000 , Li et al. (2002) showed that the magnitude of adverse pressure gradient over the suction surface near the leading edge is increased with a $2 \%$ chord Gurney flap. This may provide an explanation for an early laminar separation and a transition with plasma control. It has been shown that the air is drawn from the suction (upper) surface by the virtual Gurney flap (see figure 10), increasing the velocity near the trailing edge (see figure 8). This results in a reduction in the magnitude of adverse pressure gradient near the trailing edge, which helps the separated flow reattach earlier at $\alpha=6^{\circ}$ (Horton 1969).

\subsection{Dynamics of separation bubbles}

Figure 14 is a snapshot of spanwise vorticity in the separated shear layer over the airfoil at $\alpha=6^{\circ}$. The $\mathrm{K}-\mathrm{H}$ instability in the separated shear layer leads to a roll-up of vortices, which are then merged and convected downstream. As the separated shear layer undergoes laminar-to-turbulent transition during the vortex formation process, the vortices seem to lose coherence quickly (Yarusevych et al. 2009).

The probability density function (PDF) of the instantaneous separated shear layer centre at $\alpha=6^{\circ}$ is shown in figure 15. Here, the separated shear layer centre was determined as the location of the minimum spanwise vorticity based on 2000 snapshots. It shows that the separated shear layer for the control case is located slightly further away from the airfoil suction surface at $x / c=-0.7$ due to the 


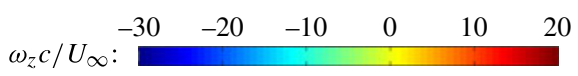

(a)

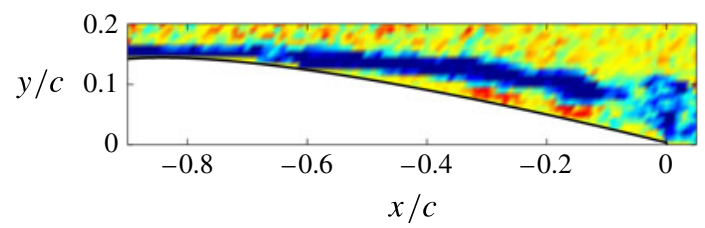

(b)

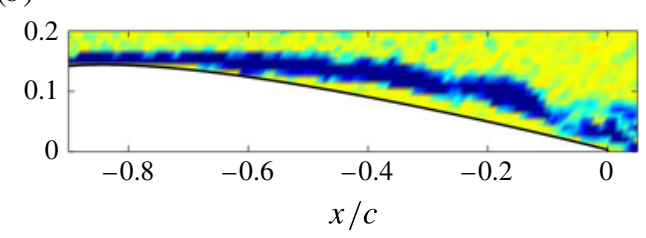

FIGURE 14. (Colour online) A snapshot of the separated shear layer over the airfoil (a) without and (b) with plasma control at $\alpha=6^{\circ}, R e=20000, C_{\mu}=1.9 \%$.

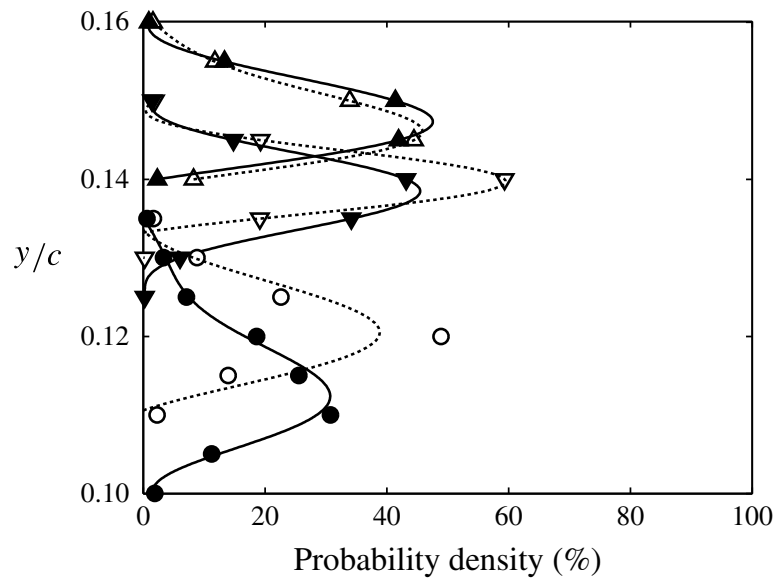

FIGURE 15. Probability density function (PDF) of the instantaneous separated shear layer centre over the airfoil suction (upper) surface without and with plasma control at $\alpha=6^{\circ}$, $R e=20000, C_{\mu}=1.9 \%: \Delta, \Delta, x / c=-0.7 ; \nabla, \nabla, x / c=-0.5 ; \bigcirc, \bullet, x / c=-0.3$. The open circles with dashed line and the filled circles with solid line denote cases without and with plasma control, respectively. There are only a few points to show the PDF because the shear layer is very thin in this condition.

advancement of the separation point. The PDFs at $x / c=-0.5$ and -0.3 are shifted closer to the airfoil surface due to early reattachment of the separated flow by the virtual Gurney flap.

The separated shear layer goes through the $\mathrm{K}-\mathrm{H}$ instability, exhibiting large fluctuation of the separated shear layer centre. The standard deviations of the vertical fluctuation of the separated shear layer centre are shown in figure 16. For cases both without and with control at $\alpha=6^{\circ}$, the standard deviation starts to increase from about $x / c=-0.6$ due to the growth of the separated shear layer and the resulting vortex shedding. The standard deviation for the control case is greater than the natural case before $x / c=-0.16$ because of the accelerated transition process with plasma control. As a result, the separated shear layer with virtual Gurney flap starts to undergo the instability process further upstream than the natural case. After that, the standard deviation for the natural case becomes much greater. The data for $\alpha=8^{\circ}$ also show a similar behaviour, suggesting that the transition is accelerated by plasma. Little difference is exhibited for $\alpha=10^{\circ}$, however. 


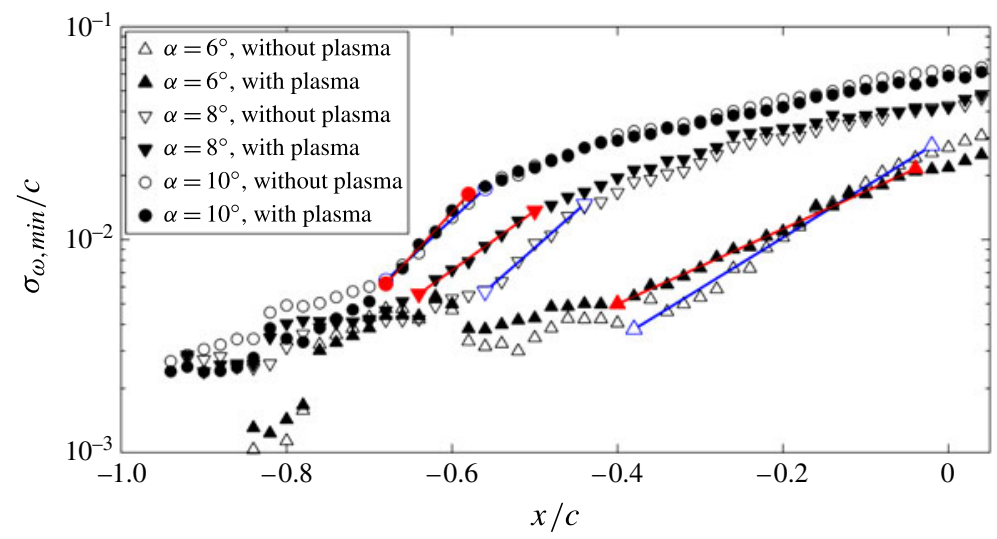

FIgURE 16. (Colour online) Standard deviation of the vertical fluctuation of the instantaneous separated shear layer centre over the airfoil suction (upper) surface at $R e=$ $20000, C_{\mu}=1.9 \%$. The open and filled symbols denote cases without and with plasma control, respectively. Straight lines in blue and red denote the exponential growth for cases without and with plasma control, respectively, where the start and the end of the exponential growth are marked.

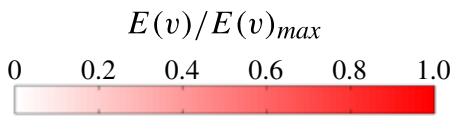

(a)

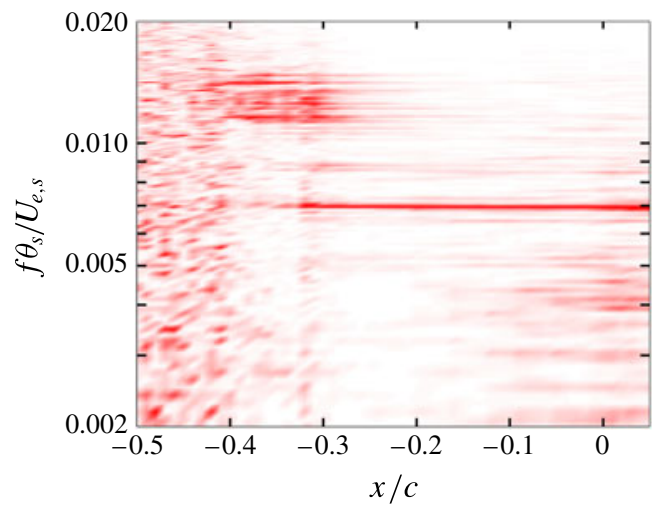

(b)

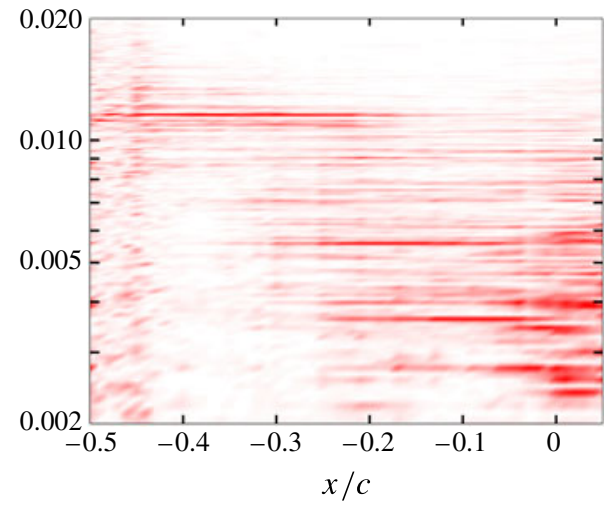

FIgURE 17. (Colour online) Contour map of the power spectrum of the vertical velocity fluctuation along the mean separated shear layer centre over the airfoil suction (upper) surface (a) without and $(b)$ with plasma control at $\alpha=6^{\circ}, R e=20000, C_{\mu}=1.9 \%$. The power spectrum at each position has been normalized by its peak.

Although the separated shear layer is highly unsteady, it still displays quasi-periodic characteristics, as indicated by the contour map of the power spectrum shown in figure 17. For the natural case, figure 17(a) illustrates a dominant frequency of $f=$ $133 \mathrm{~Hz}$ from around $x / c=-0.4$ to -0.33 , corresponding to the Strouhal number $S t=f \theta_{s} / U_{e, s}=0.0141$ based on the momentum thickness $\theta_{s}$ and the edge velocity $U_{e, s}$ at the separation point, which is considered as the $\mathrm{K}-\mathrm{H}$ instability frequency of the shear layer for the vortex roll-up. Downstream of $x / c=-0.32$, it shows a reduced 


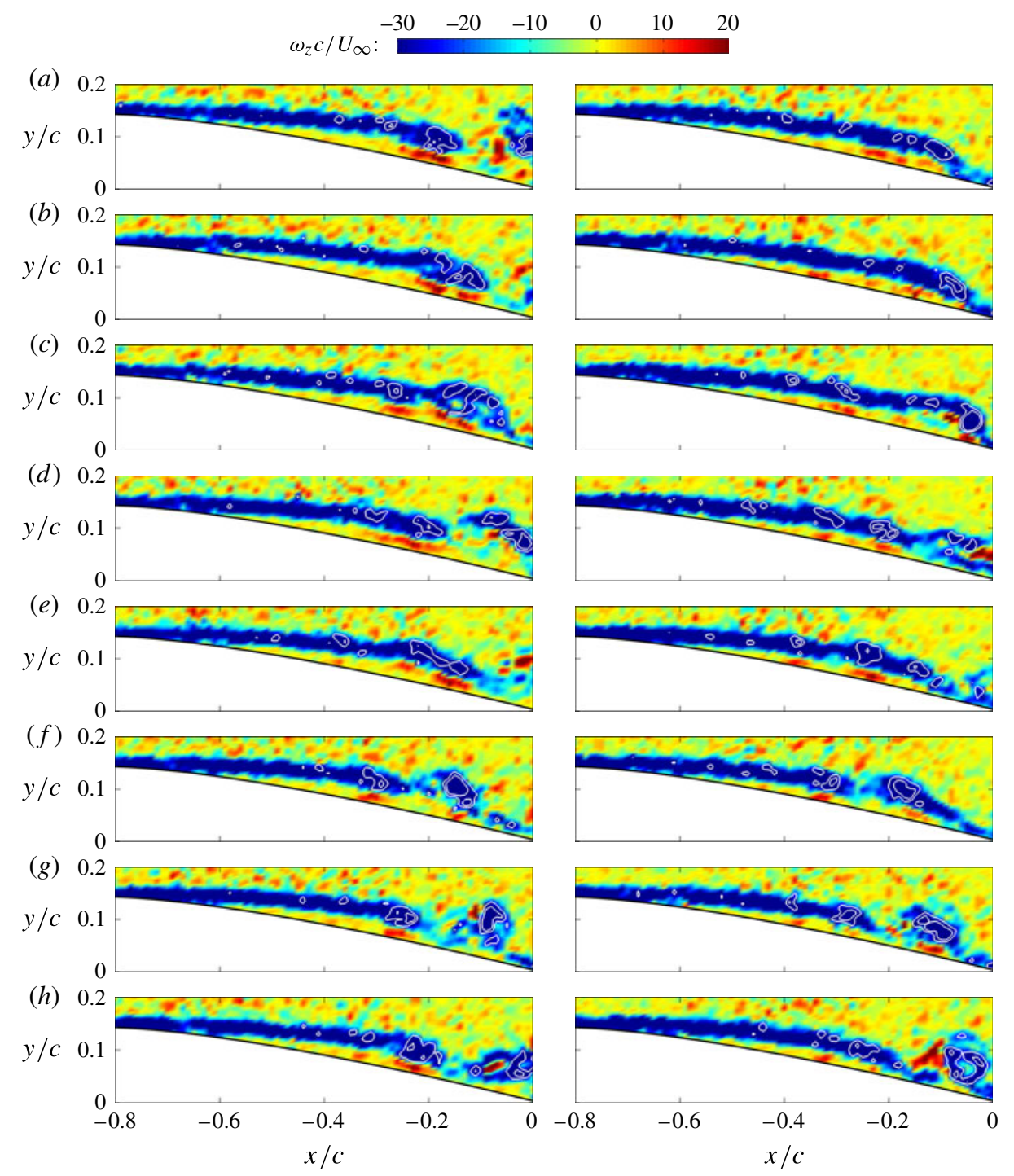

FIGURE 18. (Colour online) Instantaneous flow patterns to show the vortex merging over the airfoil without (left-hand column) and with (right-hand column) plasma control at $\alpha=$ $6^{\circ}, R e=20000, C_{\mu}=1.9 \%$. The phase information can be found in figure 19 . The figures show contour plots of the spanwise vorticity and $\lambda_{c i}$ isolines with $\lambda_{c i} c / U_{\infty}=-20,-10$.

frequency of $66 \mathrm{~Hz}(S t=0.0070)$, a sub-harmonic of the upstream K-H instability frequency until $x / c=0.05$. Such variation in the dominant frequency suggests the occurrence of the vortex-merging phenomenon, which is to be further discussed later in figure 18 . The present value $(S t=0.0070)$ of the $\mathrm{K}-\mathrm{H}$ instability frequency is consistent with the value of $S t=0.0075$ obtained by Burgmann \& Schröder (2008) for the flow over an SD7003 airfoil at $\alpha=6^{\circ}, R e=19900$. However, they did not observe the vortex-merging phenomenon, therefore they were not able to show the harmonic value of $S t=0.0141$ found in this study. With plasma control (figure $17 b$ ), the $\mathrm{K}-\mathrm{H}$ instability frequency of the shear layer stays the same at $133 \mathrm{~Hz}(\mathrm{St}=0.0115)$ from 

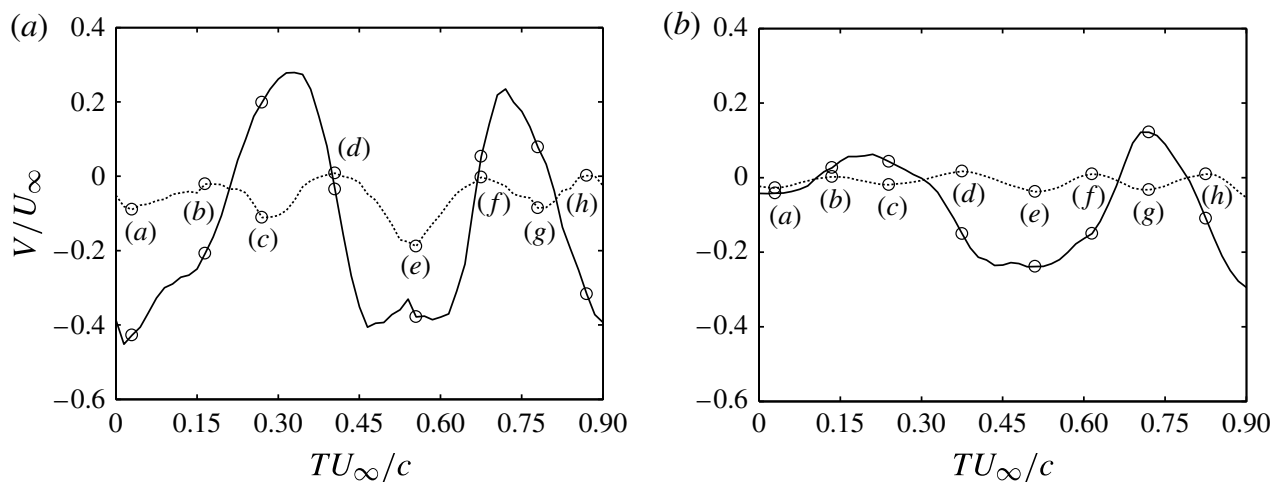

FIGURE 19. Time evolution of the vertical velocity at the separated shear layer centre at $x / c=-0.35$ (dashed line) and -0.15 (solid line) for the airfoil, $(a)$ without and $(b)$ with plasma control at $\alpha=6^{\circ}, R e=20000, C_{\mu}=1.9 \%$; $(a)-(h)$ denote the phase angle of the instantaneous flow patterns shown in figure 18.

$x / c=-0.48$ to -0.22 , which is reduced to its sub-harmonic of $65 \mathrm{~Hz}(S t=0.0056)$ from $x / c=-0.21$ to -0.14 . However, the rolled-up vortices lose their dominant frequency rapidly as they are shed downstream. It is shown that the peaks of the power spectrum downstream of $x / c=-0.25$ vary around $S t=0.0036$, while there is also a local peak around $S t=0.0027$. These values agree well with the bi-modal vortex shedding observed by Troolin et al. (2006) for the airfoil with a mechanical Gurney flap. This provides additional evidence that the virtual Gurney flap creates a recirculation region similar to that of the mechanical Gurney flap.

The streamwise change in the dominant shedding frequency can indicate the dynamic behaviour of the vortical structures during their formation and shedding process. An example is given in figure 18 to show the evolution of instantaneous spanwise vorticity superposed by the $\lambda_{c i}$ isolines over the airfoil without and with plasma control at $\alpha=6^{\circ}$, where the corresponding phase information can be found in figure 19. Here, $\lambda_{c i}$ is the imaginary part of the complex eigenvalue of the velocity gradient tensor (Zhou et al. 1999), which has the same sign as the local spanwise vorticity. For the natural case, small-scale vortices can be observed at $x / c=-0.4$, which become greater downstream. Often there are two adjacent vortices formed one after the other, as shown in figure $18(c, d)$ in a region between $x / c=-0.4$ and -0.2 . They merge at around $x / c=-0.2$ as shown in figure 18(e) to form a larger-scale vortical structure. The merged vortex starts to move away from the main shear layer to be shed downstream. The variation of the vertical velocity at $x / c=-0.35$ and -0.15 shown in figure $19(a)$ is a result of this vortex-merging process, indicating that the period of the velocity at $x / c=-0.15$ is increased to twice that at $x / c=-0.35$. With plasma control, a similar vortex-merging process is observed, which can be observed just downstream of $x / c=-0.2$, as shown in figure $18(f)$. The vertical velocity variation shown in figure $19(b)$ confirms that the vortex formation frequency at $x / c=-0.15$ is a sub-harmonic of the frequency at $x / c=-0.35$. The vortex-merging process over an airfoil for both cases can be clearly seen in figure 20 in the time history of $\lambda_{c i} c / U_{\infty}$ along the separated shear layer centre.

Figure 21 shows the phase-averaged spanwise vorticity at $\alpha=6^{\circ}$, which is superposed by streamlines and the $\lambda_{c i}$ isolines. The separation bubbles were detected from the streamlines of this figure, while the vortical structure was identified by 


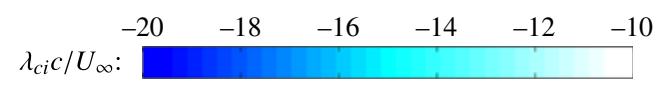

(a)

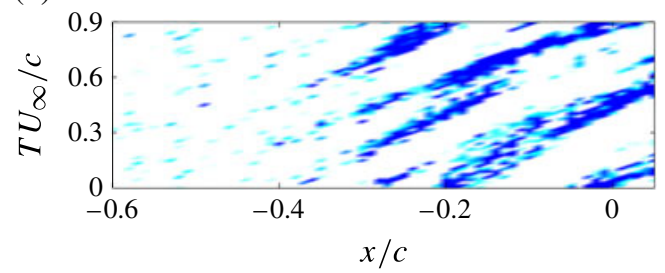

(b)

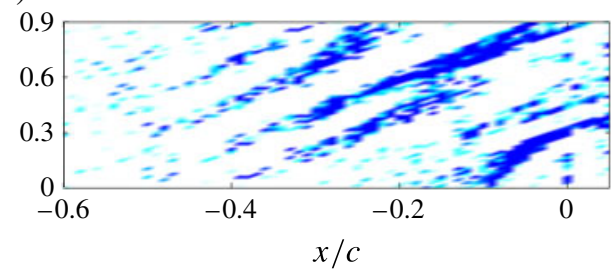

FIgURE 20. (Colour online) Time history of $\lambda_{c i} c / U_{\infty}$ along the separated shear layer centre for the airfoil, (a) without and (b) with plasma control at $\alpha=6^{\circ}, R e=20000$, $C_{\mu}=1.9 \%$.

the $\lambda_{c i}$ contours. As has been shown above, there is no unique dominant frequency during the vortex evolution along the airfoil suction surface. Thus, reference velocity signals at $x / c=-0.35$ and -0.15 were used to performance the phase average of the flow field over the airfoil. Figure 21(a,c,e,g,i,k,m,o) shows a periodic formation of the vortical structure in a region between $x / c=-0.4$ and -0.3 , while the vortex-merging process can be observed at around $x / c=-0.2$. Figure $21(b, d, f, h, j, l, n, p)$, on the other hand, shows a process of periodic merging and shedding of vortices with an increase in the concentrated vorticity within. The dynamic behaviour of the separation bubble depicted in figure 21 is very similar to that observed by Pauley et al. (1990), Burgmann \& Schröder (2008) and Postl et al. (2011).

Figure 22 shows the phase-averaged flow field over the airfoil with plasma control at $\alpha=6^{\circ}, R e=20000, C_{\mu}=1.9 \%$. Figure $22(a, c, e, g, i, k, m, o)$ demonstrates that the periodic formation of vortices starts further upstream with plasma control. This is due to the advancement of the flow separation point and the start of transition with plasma control. Figure $22(b, d, f, h, j, l, n, p)$ shows the merging and shedding of vortices at around $x / c=-0.15$. It shows that the shed vortex loses its coherence more rapidly than in the natural case. This is also evident in the vorticity map (figures 9 and 10) as well as in the power spectrum in figure 17.

The dynamics of separation bubbles and the associated vortical structure in the separated shear layer are shown in figure 23. Here, the separation bubble centre was obtained by detecting the focus of streamlines that spiralled in (see figures $21 b, d, f, h, j, l, n, p$ and $22 b, d, f, h, j, l, n, p)$, while the vortex centre was obtained by finding the local maximum $\lambda_{c i}$ value. Figure 23(a) shows that the separation bubble and the vortical structure move downstream over the airfoil surface for four continuous periods, from phase $\Phi=0^{\circ}$ to $1440^{\circ}$. The convection velocity of the separation bubble and the associated vortical structure can be obtained from the slope of the phase evolution in figure 23(a). The convection velocity of the separation bubble is $0.36 U_{\infty}$ for the natural case, while it is slightly less at $0.34 U_{\infty}$ with plasma control. The convection velocity of the vortical structure within the separated shear layer is greater than that of the separation bubble, which is $0.43 U_{\infty}$ and $0.37 U_{\infty}$ for the natural and the control cases, respectively. The convection velocity of the vortical structure in our experiment compares well with the value of $0.43 U_{\infty}$ obtained by Saathoff \& Melbourne (1997) over a flat plate with a blunt leading edge and with the value of $0.5 U_{\infty}$ obtained by Kiya \& Sasaki (1983) and Sung, Chung \& Kiya (1996). 


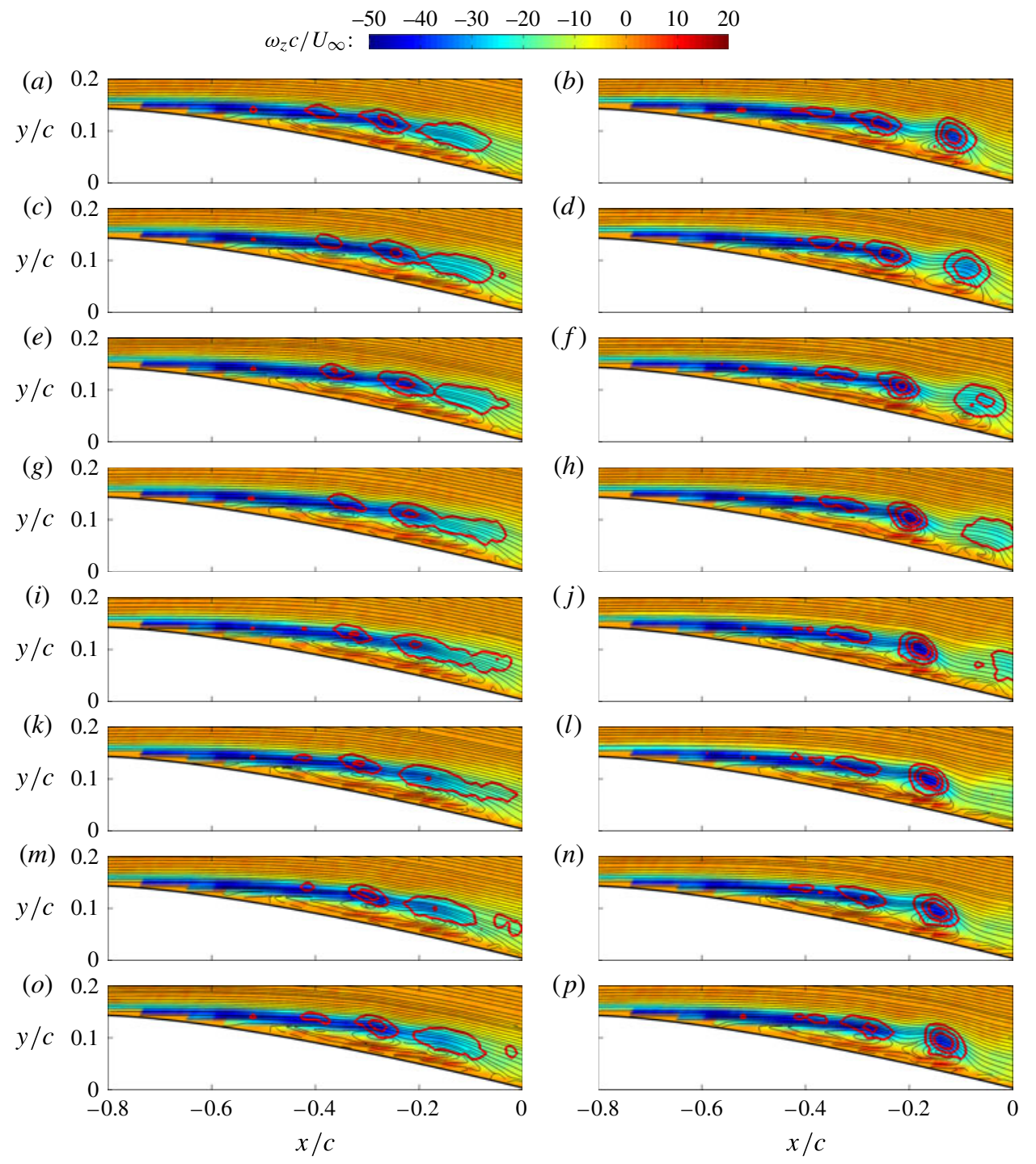

FIgURE 21. (Colour online) Phase evolution of the shear layer and the separation bubble at eight phases: $(a, b) \Phi=0^{\circ},(c, d) \Phi=45^{\circ},(e, f) \Phi=90^{\circ},(g, h) \Phi=135^{\circ},(i, j) \Phi=180^{\circ}$, $(k, l) \Phi=225^{\circ},(m, n) \Phi=270^{\circ},(o, p) \Phi=315^{\circ}$, for the airfoil without plasma control at $\alpha=6^{\circ}, R e=20000$. The phase-averaging patterns in $(a, c, e, g, i, k, m, o)$ are obtained based on the vertical velocity signal in the shear layer region at $x / c=-0.35$ and those in $(b, d, f, h, j, l, n, p)$ at $x / c=-0.15$. The figures show contour plots of the spanwise vorticity superposed with the streamlines and $\lambda_{c i}$ isolines with $\lambda_{c i} c / U_{\infty}=-12,-8,-4$.

Figure 23(b) together with figures 21 and 22 indicate the locations of the separation bubble centre and the vortical structure centre relative to the airfoil surface, showing that the vortices are located just outside of the separation bubbles. It is shown that both the separation bubble centre and the vortex centre are located closer to the suction surface of the airfoil with plasma control, which is consistent with the results in figure 15. 


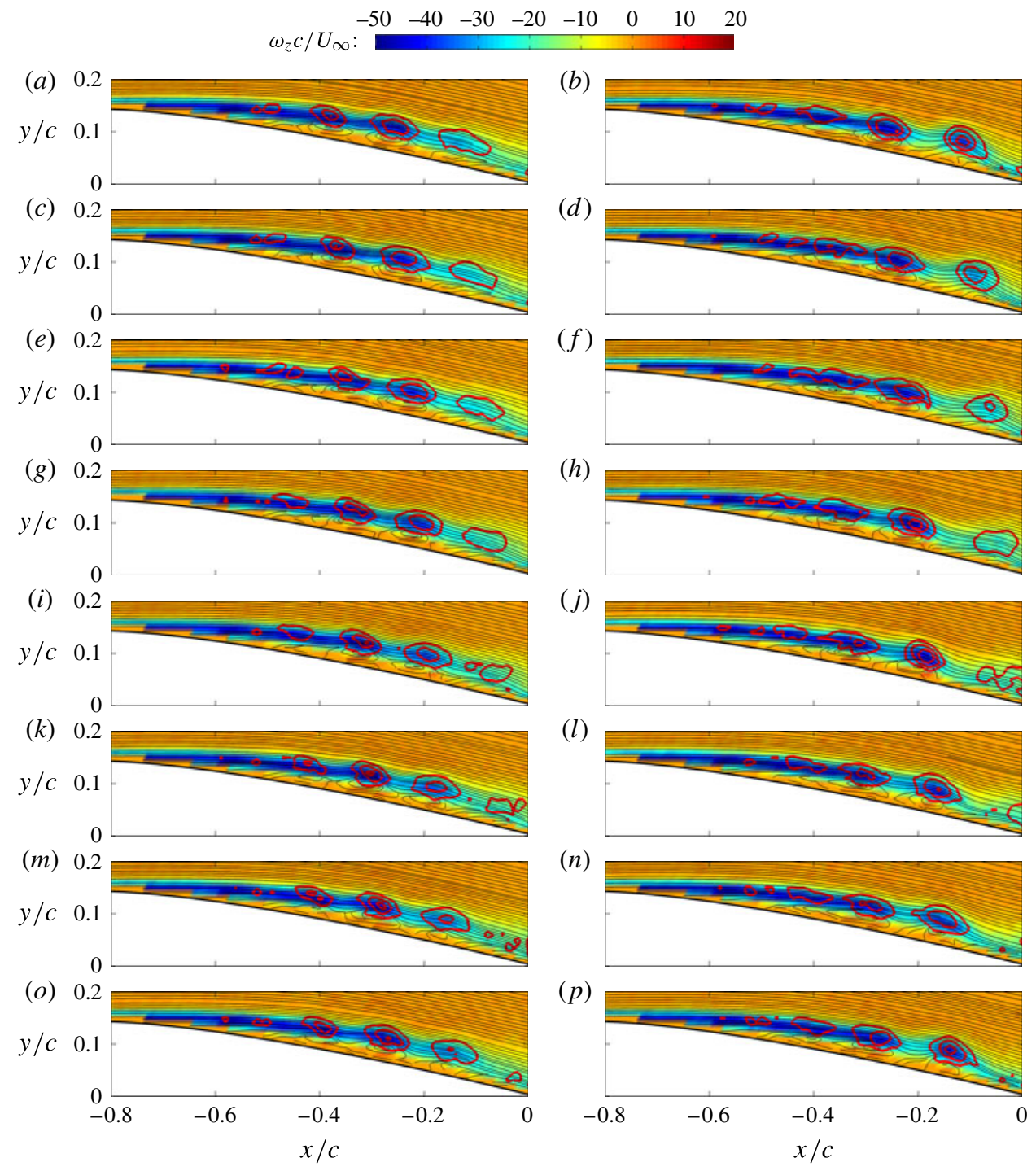

FIGURE 22. (Colour online) Phase evolution of the shear layer and the separation bubble at eight phases: $(a, b) \Phi=0^{\circ},(c, d) \Phi=45^{\circ},(e, f) \Phi=90^{\circ},(g, h) \Phi=135^{\circ},(i, j) \Phi=180^{\circ}$, $(k, l) \Phi=225^{\circ},(m, n) \Phi=270^{\circ},(o, p) \Phi=315^{\circ}$, for the airfoil with plasma control at $\alpha=$ $6^{\circ}, R e=20000, C_{\mu}=1.9 \%$. The phase-averaging patterns in $(a, c, e, g, i, k, m, o)$ are obtained based on the vertical velocity signal in the shear layer region at $x / c=-0.35$ and those in $(b, d, f, h, j, l, n, p)$ at $x / c=-0.15$. These figures show contour plots of the spanwise vorticity superposed with the streamlines and $\lambda_{c i}$ isolines with $\lambda_{c i} c / U_{\infty}=-12,-8,-4$.

\section{Conclusions}

Flow control over a NACA 0012 airfoil is carried out using a DBD plasma actuator at a Reynolds number of 20000 . Here, the plasma actuator is placed over the pressure side of the airfoil near the trailing edge, which produces a wall jet against the free stream to form a quasi-steady recirculation region. This results in a reduction in the velocity over the pressure surface of the airfoil. This recirculation region also 

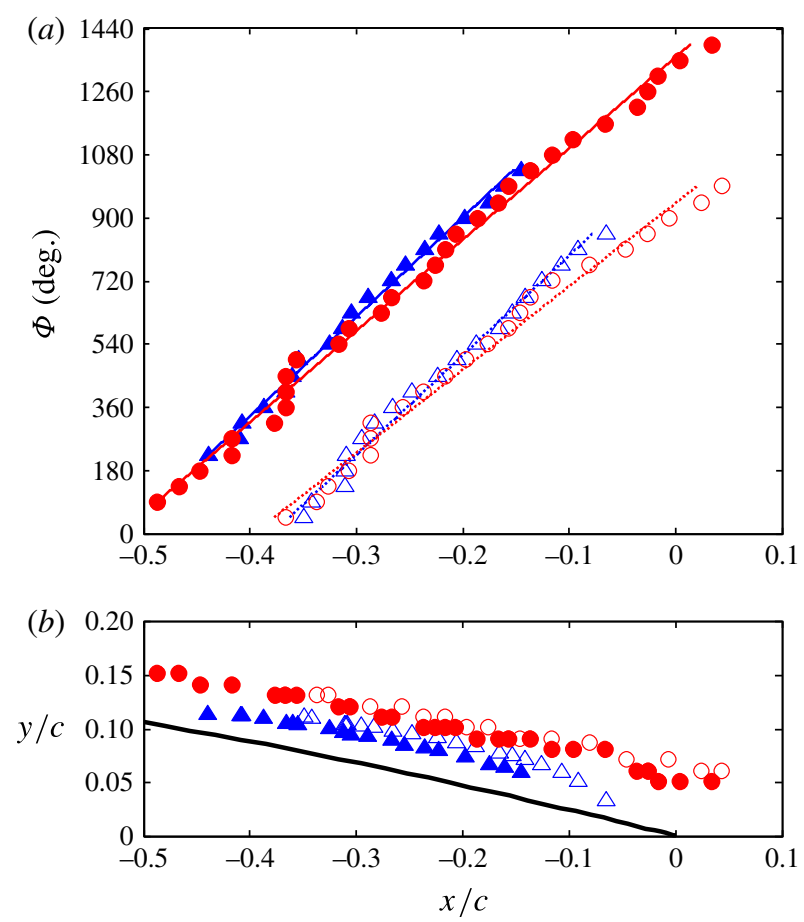

Figure 23. (Colour online) Phase evolution of the separation bubble centre $(\Delta, \mathbf{\Delta})$ and the vortical structure centre $(O, \bullet)$ in $(a)$ the $x / c-\Phi$ plane and $(b)$ the $x / c-y / c$ plane at $\alpha=6^{\circ}, R e=20000, C_{\mu}=1.9 \%$. The open symbols with dashed lines and the filled symbols with solid lines denote cases without and with plasma control, respectively, where the straight lines show the linear fitting curves for the corresponding cases.

draws the air over the suction surface toward the trailing edge, helping to reattach the separated flow. These flow modifications around the airfoil lead to an increase in the pressure difference between the pressure and suction surfaces, resulting in an increase in the lift coefficient. The maximum lift enhancement with plasma control is $23 \%$ at the plasma jet momentum coefficient of $C_{\mu}=1.9 \%$. Our results suggest that the mechanism involved in lift enhancement using this type of DBD plasma actuator is very similar to that of mechanical Gurney flaps. We therefore call them virtual Gurney flaps. It is shown that the plasma forcing of a virtual Gurney flap with $C_{\mu}=1 \%$ is equivalent to a mechanical Gurney flap of $1 \%$ chord length in terms of lift enhancement.

Measured phase-averaged vorticity and velocity fields around the airfoil indicate that the recirculation region created by the virtual Gurney flap over the pressure surface modifies the near-wake dynamics. Firstly, the recirculation region draws the air from the suction surface around the trailing edge, thereby enhancing the negative vorticity layer from the suction surface. The upper shear layer then interacts with the opposite-signed shear layer from the pressure surface, creating a stronger vortex shedding from the airfoil. This is an important ingredient for an effective, active flow control system for lift enhancement (Colonius \& Williams 2011). Secondly, the recirculation region created by a DBD plasma actuator over the pressure surface displaces the positive shear layer away from the airfoil, thereby shifting the near-wake 
region downwards. This leads to bending of the streamlines around the trailing edge, effectively creating a camber on the airfoil. This results in a lift coefficient of approximately 0.2 at zero angle of attack when the plasma actuator is activated with the jet momentum coefficient of $C_{\mu}=1.9 \%$.

There is an initial development of T-S waves over the airfoil, followed by a growth of $\mathrm{K}-\mathrm{H}$ instability. The dynamics of laminar separation bubbles and associated vortical structures changes with plasma control due to the accelerated laminar-to-turbulent transition through the $\mathrm{K}-\mathrm{H}$ instability mechanism. In particular, the separation point is moved upstream by $\Delta x_{s} / c=0.04,0.04$ and 0.01 at angles of attack $\alpha=6^{\circ}, 8^{\circ}$ and $10^{\circ}$, respectively, while the start of transition is advanced by $\Delta x_{s t} / c=0.06$, 0.01 and 0.01 . The reattachment point also moves upstream with plasma control by $\Delta x_{r} / c=0.12$ and 0.02 at $\alpha=6^{\circ}$ and $8^{\circ}$, respectively, although it is delayed by $\Delta x_{r} / c=0.05$ at $\alpha=10^{\circ}$. The shed vortices lose their coherence much more rapidly than in the natural case, as the virtual Gurney flap creates a bi-modal shedding frequency similar to the mechanical Gurney flap.

\section{Acknowledgements}

Gratitude is due to the China Scholarship Council and the Royal Academy of Engineering for supporting this joint research programme. We would also like to acknowledge EPSRC Research Grant EP/G025150/1, under which the PIV system that was used in this study was purchased. The first author would like to thank the support of Specialized Research Fund for the Doctoral Program of Higher Education (no. 20121102120015) and the Fundamental Research Funds for the Central Universities (no. YWF-14-HKXY-007) in conducting this study.

\section{REFERENCES}

Alam, M. \& SAndham, N. D. 2000 Direct numerical simulation of 'short' laminar separation bubbles with turbulent reattachment. J. Fluid Mech. 410, 1-28.

Almutairi, J. H., Jones, L. E. \& SAndham, N. D. 2010 Intermittent bursting of a laminar separation bubble on an airfoil. AIAA J. 48, 414-426.

Atik, H., Kim, C. Y., VAn Dommelen, L. L. \& Walker, J. D. A. 2005 Boundary-layer separation control on a thin airfoil using local suction. J. Fluid Mech. 535, 415-443.

BAtill, S. M. \& Muller, T. J. 1981 Visualization of transition in the flow over an airfoil using the smoke-wire technique. AIAA J. 19, 340-345.

Benard, N., Jolibois, J. \& Moreau, E. 2009 Lift and drag performances of an axisymmetric airfoil controlled by plasma actuator. J. Electrostat. 67, 133-139.

Boutilier, M. S. H. \& YARUSEvyCH, S. 2012 Separated shear layer transition over an airfoil at a low Reynolds number. Phys. Fluids 24, 084105.

BurgmanN, S., BRÜCKER, C. \& SCHRÖDER, W. 2006 Scanning PIV measurements of a laminar separation bubble. Exp. Fluids 41, 319-326.

Burgmann, S., DAnnemann, J. \& SChröDer, W. 2008 Time-resolved and volumetric PIV measurements of a transitional separation bubble on an SD7003 airfoil. Exp. Fluids 44, 609-622.

BURGMANN, S. \& SCHRÖDER, W. 2008 Investigation of the vortex induced unsteadiness of a separation bubble via time-resolved and scanning PIV measurements. Exp. Fluids 45, 675-691.

Colonius, T. \& Williams, D. R. 2011 Control of vortex shedding on two- and three-dimensional aerofoils. Phil. Trans. R. Soc. A 369, 1525-1539.

Corke, T. C., Enloe, C. L. \& Wilkinson, S. P. 2010 Dielectric barrier discharge plasma actuators for flow control. Annu. Rev. Fluid Mech. 42, 505-529. 
Diwan, S. S. \& RAmesh, O. N. 2009 On the origin of the inflectional instability of a laminar separation bubble. J. Fluid Mech. 629, 263-298.

Duvigneau, R., Hay, A. \& Visonneau, M. 2007 Optimal location of a synthetic jet on an airfoil for stall control. Trans. ASME: J. Fluids Engng 129, 825-833.

Ellsworth, R. H. \& Mueller, T. J. 1991 Airfoil boundary layer measurements at low Re in an accelerating flow from a nonzero velocity. Exp. Fluids 11, 368-374.

FenG, L. H., Jukes, T. N., ChOI, K. S. \& WANG, J. J. 2012 Flow control over a NACA 0012 airfoil using dielectric-barrier-discharge plasma actuator with a Gurney flap. Exp. Fluids 52, $1533-1546$.

FEnG, L. H. \& WANG, J. J. 2010 Circular cylinder vortex-synchronization control with a synthetic jet positioned at the rear stagnation point. J. Fluid Mech. 662, 232-259.

GASTER, M. 1967 The structure and behaviour of laminar separation bubbles. Aero. Res. Counc. R \& $M$ 3595, pp. 1-31.

Genç, M. S., KAYNAK, Ü. \& YAPICI, H. 2011 Performance of transition model for predicting low $\mathrm{Re}$ aerofoil flows without/with single and simultaneous blowing and suction. Eur. J. Mech. (B/Fluids) 30, 218-235.

Greenblatt, D. \& Wygnanski, I. J. 2000 The control of flow separation by periodic excitation. Prog. Aerosp. Sci. 36, 487-545.

HAin, R., KähleR, C. J. \& RAdespiel, R. 2009 Dynamics of laminar separation bubbles at low-Reynolds-number aerofoils. J. Fluid Mech. 630, 129-153.

Hatman, A. \& WANG, T. 1999 A prediction model for separated-flow transition. Trans. ASME: J. Turbomach. 121, 594-602.

He, C., Corke, T. C. \& Patel, M. P. 2009 Plasma flaps and slats: an application of weakly ionized plasma actuators. J. Aircraft 46, 864-873.

Horton, H. P. 1969 A semi-empirical theory for the growth and bursting of laminar separation bubbles. Aero. Res. Counc. Current Papers 1073.

HuAng, R. F. \& Lin, C. L. 1995 Vortex shedding and shear-layer instability of wing at low Reynolds numbers. AIAA J. 33, 1398-1403.

InASAWA, A., NinOMIYA, C. \& ASAI, M. 2013 Suppression of tonal trailing-edge noise from an airfoil using a plasma actuator. AIAA J. 51, 1695-1702.

Jeffrey, D. R. M. \& Hurst, D. W. 1996 Aerodynamic of the Gurney flap. AIAA Paper 96-2418.

Jones, L. E., SANDBerg, R. D. \& SAndham, N. D. 2010 Stability and receptivity characteristics of a laminar separation bubble on an aerofoil. J. Fluid Mech. 648, 257-296.

JUKes, T. N. \& CHOI, K.-S. 2009 Flow control around a circular cylinder using pulsed dielectric barrier discharge surface plasma. Phys. Fluids 21, 084103.

Jukes, T. N., Choi, K.-S., Johnson, G. A. \& ScotT, S. J. 2006 Characterization of surface plasmainduced wall flows through velocity and temperature measurements. AIAA J. 44, 764-771.

Jukes, T. N., Choi, K.-S., Segawa, T. \& Yoshida, H. 2008 Jet flow induced by a surface plasma actuator. Proc. Inst. Mech. Engrs I 222, 347-356.

KIM, D. H., ChAng, J. W. \& ChUng, J. 2011 Low-Reynolds-number effect on aerodynamic characteristics of a NACA 0012 airfoil. J. Aircraft 48, 1212-1215.

Kinzel, M. P., Maughmer, M. D. \& Duque, E. P. N. 2010 Numerical investigation on the aerodynamics of oscillating airfoils with deployable Gurney flaps. AIAA J. 48, 1457-1469.

KIYA, M. \& SASAKI, K. 1983 Structure of a turbulent separation bubble. J. Fluid Mech. 137, 83-113.

LAitone, E. V. 1997 Wind tunnel tests of wings at Reynolds numbers below 70000. Exp. Fluids 23, 405-409.

LANG, M., Rist, U. \& Wagner, S. 2004 Investigations on controlled transition development in a laminar separation bubble by means of LDA and PIV. Exp. Fluids 36, 43-52.

LEE, T. 2009 Aerodynamic characteristics of airfoil with perforated Gurney-type flaps. J. Aircraft 46, $542-548$.

LEE, T. 2011 PIV study of near-field tip vortex behind perforated Gurney flaps. Exp. Fluids 50, 351-361.

LEE, T. \& Ko, L. S. 2009 PIV investigation of flowfield behind perforated Gurney-type flaps. Exp. Fluids 46, 1005-1019. 
LEE, T. \& SU, Y. Y. 2011 Lift enhancement and flow structure of airfoil with joint trailing-edge flap and Gurney flap. Exp. Fluids 50, 1671-1684.

LI, Y. C., WANG, J. J. \& HUA, J. 2007 Experimental investigations on the effects of divergent trailing edge and Gurney flaps on a supercritical airfoil. Aerosp. Sci. Technol. 11, 91-99.

Li, Y. C., WANG, J. J. \& ZhANG, P. F. 2002 Effect of Gurney flaps on a NACA0012 airfoil. Flow Turbul. Combust. 68, 27-39.

LI, Y. C., WANG, J. J. \& ZhANG, P. F. 2003 Influences of mounting angles and locations on the effects of Gurney flaps. J. Aircraft 40, 494-498.

LiAn, Y. \& SHYY, W. 2007 Laminar-turbulent transition of a low Reynolds number rigid or flexible airfoil. AIAA J. 45, 1501-1513.

LiebeCK, R. H. 1978 Design of subsonic airfoils for high lift. J. Aircraft 15, 547-561.

Little, J., Nishihara, M., Adamovich, I. \& Samimy, M. 2010 High-lift airfoil trailing edge separation control using a single dielectric barrier discharge plasma actuator. Exp. Fluids 48, 521-537.

Little, J. \& SAmimy, M. 2010 High-lift airfoil separation with dielectric barrier discharge plasma actuation. AIAA J. 48, 2884-2898.

LiU, T. S. \& Montefort, J. 2007 Thin-airfoil theoretical interpretation for Gurney flap lift enhancement. J. Aircraft 44, 667-671.

Lombardi, A. J., Bowles, P. O. \& Corke, T. C. 2013 Closed-loop dynamic stall control using a plasma actuator. AIAA J. 51, 1130-1141.

Mabe, J. H., Calkins, F. T., Wesley, B., Woszidlo, R., Taubert, L. \& Wygnanski, I. 2009 Single dielectric barrier discharge plasma actuators for improved airfoil performance. J. Aircraft 46, 847-855.

MARXEN, O. \& Rist, U. 2010 Mean flow deformation in a laminar separation bubble: separation and stability characteristics. J. Fluid Mech. 660, 37-54.

Moreau, E. 2007 Airflow control by non-thermal plasma actuators. J. Phys. D: Appl. Phys. 40, 605-636.

Mueller, T. J. \& Delaurier, J. D. 2003 Aerodynamics of small vehicles. Annu. Rev. Fluid Mech. 35, 89-111.

Nickerson, J. D. 1986 A study of vortex generators at low Reynolds numbers. AIAA Paper 1986-0155.

O'Meara, M. M. \& Mueller, T. J. 1987 Laminar separation bubble characteristics on an airfoil at low Reynolds numbers. AIAA J. 25, 1033-1041.

Pauley, L. L., Moin, P. \& Reynolds, W. C. 1990 The structure of two-dimensional separation. J. Fluid Mech. 220, 397-411.

Post, M. L. \& CoRKe, T. C. 2004 Separation control on high angle of attack airfoil using plasma actuators. AIAA J. 42, 2177-2184.

Post, M. L. \& CORKE, T. C. 2006 Separation control using plasma actuators: dynamic stall vortex control on oscillating airfoil. AIAA J. 44, 3125-3135.

Postl, D., BAlzer, W. \& FASEL, H. F. 2011 Control of laminar separation using pulsed vortex generator jets: direct numerical simulations. J. Fluid Mech. 676, 81-109.

Rist, U. \& Augustin, K. 2006 Control of laminar separation bubbles using instability waves. AIAA J. 44, 2217-2223.

Rizzetta, D. P. \& Visbal, M. R. 2011 Numerical investigation of plasma-based control for lowReynolds-number airfoil flows. AIAA J. 49, 411-425.

Rizzetta, D. P. \& Visbal, M. R. 2012 Effect of plasma-based control on low-Reynolds-number flapping airfoil performance. AIAA J. 50, 131-147.

Rogers, E. O. \& Donnelly, M. J. 2004 Characteristics of a dual-slotted circulation control wing of low aspect ratio intended for naval hydrodynamic applications. AIAA Paper 2004-1244.

SaAthoff, P. J. \& Melbourne, W. H. 1997 Effects of free-stream turbulence on surface pressure fluctuations in a separation bubble. J. Fluid Mech. 337, 1-24.

Schuele, C. Y. \& Greenblatt, D. 2010 Combined plasma and Gurney flap flow control at low flight Reynolds numbers. AIAA J. 48, 2714-2718. 
Seshagiri, A., Cooper, E. \& Traub, L. W. 2009 Effects of vortex generators on an airfoil at low Reynolds numbers. J. Aircraft 46, 116-122.

Singh, M. K., Dhanalakshmi, K. \& ChakrabartTy, S. K. 2007 Navier-Stokes analysis of airfoils with Gurney flap. J. Aircraft 44, 1487-1493.

Sosa, R., Artana, G., Moreau, E. \& Touchard, G. 2007 Stall control at high angle of attack with plasma sheet actuators. Exp. Fluids 42, 143-167.

Spalart, P. R. \& STRelets, M. K. 2000 Mechanisms of transition and heat transfer in a separation bubble. J. Fluid Mech. 403, 329-349.

Sung, H. J., Chung, Y. M. \& KIYA, M. 1996 Vortex simulation of leading-edge separation bubble with local forcing. Fluid Dyn. Res. 18, 99-115.

Traub, L. W. \& Agarwal, G. 2008 Aerodynamic characteristics of a Gurney/jet flap at low Reynolds numbers. J. Aircraft 45, 424-429.

Traub, L. W., Miller, A. C. \& Rediniotis, O. 2004 Comparisons of a Gurney and jet flap for hingeless control. J. Aircraft 41, 420-423.

Troolin, D. R., Longmire, E. K. \& LAI, W. T. 2006 Time resolved PIV analysis of flow over a NACA0015 airfoil with Gurney flap. Exp. Fluids 41, 241-254.

Vorobiev, A., Rennie, R. M. \& Jumper, E. J. 2013 Lift enhancement by plasma actuators at low Reynolds numbers. J. Aircraft 50, 12-19.

WAHIDI, R. \& BRIDGES, D. H. 2012 Effects of distributed suction on an airfoil at low Reynolds number. AIAA J. 50, 523-539.

Wang, J. J., Choi, K.-S., Feng, L. H., Jukes, T. N. \& Whalley, R. D. 2013 Recent developments in DBD plasma flow control. Prog. Aerosp. Sci. 62, 52-78.

WANG, J. J., LI, Y. C. \& CHOI, K.-S. 2008 Gurney flap-lift enhancement, mechanisms and applications. Prog. Aerosp. Sci. 44, 22-47.

WATMUfF, J. H. 1999 Evolution of a wave packet into vortex loops in a laminar separation bubble. J. Fluid Mech. 397, 119-169.

Wei, Q. K., Niu, Z. G., Chen, B. \& HuAng, X. 2013 Bang-bang control applied in airfoil roll control with plasma actuators. J. Aircraft 50, 670-676.

Wu, J. Z., Lu, X. Y., Denny, A. G., FAn, M. \& WU, J. M. 1998 Post-stall flow control on an airfoil by local unsteady forcing. J. Fluid Mech. 371, 21-58.

YANG, Z. \& HU, H. 2008 Laminar flow separation and transition on a low-Reynolds-number airfoil. J. Aircraft 45, 1067-1070.

YARUSEVyCh, S. \& Boutilier, M. S. H. 2011 Vortex shedding of an airfoil at low Reynolds numbers. AIAA J. 49, 2221-2227.

Yarusevych, S., Sullivan, P. E. \& Kawall, J. G. 2005 Airfoil boundary layer separation and control at low Reynolds numbers. Exp. Fluids 38, 545-547.

Yarusevych, S., Sullivan, P. E. \& Kawall, J. G. 2006 Coherent structures in an airfoil boundary layer and wake at low Reynolds numbers. Phys. Fluids 18, 044101.

Yarusevych, S., Sullivan, P. E. \& Kawall, J. G. 2009 On vortex shedding from an airfoil in low-Reynolds-number flows. J. Fluid Mech. 632, 245-271.

YU, T., WANG, J. J. \& ZHANG, P. F. 2011 Numerical simulation of Gurney flap on RAE-2822 supercritical airfoil. J. Aircraft 48, 1565-1575.

Zaman, K. B. M. Q., BAR-Severs, A. \& Mangalam, S. M. 1987 Effect of acoustic excitation on the flow over a low-Re airfoil. J. Fluid Mech. 182, 127-148.

ZhANG, P. F., LIU, A. B.\& WANG, J. J. 2009 Aerodynamic modification of a NACA 0012 airfoil by trailing-edge plasma Gurney flap. AIAA J. 47, 2467-2474.

ZhANG, P. F., YAN, B., LIU, A. B. \& WANG, J. J. 2010 Numerical simulation on plasma circulation control airfoil. AIAA J. 48, 2213-2225.

Zhou, J., Adrian, R. J., Balachandar, S. \& Kendall, T. M. 1999 Mechanisms for generating coherent packets of hairpin vortices in channel flow. J. Fluid Mech. 387, 353-396.

Zhou, Y., Alam, M. M., Yang, H. X., Guo, H.\& Wood, D. H. 2011 Fluid forces on a very low Reynolds number airfoil and their prediction. Intl J. Heat Fluid Flow 32, 329-339. 